\title{
Geometry and violent events in turbulent pair dispersion
}

\author{
Rehab Bitane, Holger Homann, and Jérémie Bec* \\ Laboratoire J.-L. Lagrange UMR 7293, Université de Nice-Sophia Antipolis, CNRS, \\ Observatoire de la Côte d'Azur, BP 4229, 06304 Nice Cedex 4, France
}

(November 16, 2018)

\begin{abstract}
The statistics of Lagrangian pair dispersion in a homogeneous isotropic flow is investigated by means of direct numerical simulations. The focus is on deviations from Richardson eddydiffusivity model and in particular on the strong fluctuations experienced by tracers. Evidence is obtained that the distribution of distances attains an almost self-similar regime characterized by a very weak intermittency. The timescale of convergence to this behavior is found to be given by the kinetic energy dissipation time measured at the scale of the initial separation. Conversely the velocity differences between tracers are displaying a strongly anomalous behavior whose scaling properties are very close to that of Lagrangian structure functions. These violent fluctuations are interpreted geometrically and are shown to be responsible for a long-term memory of the initial separation. Despite this strong intermittency, it is found that the mixed moment defined by the ratio between the cube of the longitudinal velocity difference and the distance attains a statistically stationary regime on very short timescales. These results are brought together to address the question of violent events in the distribution of distances. It is found that distances much larger than the average are reached by pairs that have always separated faster since the initial time. They contribute a stretched exponential behavior in the tail of the inter-tracer distance probability distribution. The tail approaches a pure exponential at large times, contradicting Richardson diffusive approach. At the same time, the distance distribution displays a time-dependent power-law behavior at very small values, which is interpreted in terms of fractal geometry. It is argued and demonstrated numerically that the exponent converges to one at large time, again in conflict with Richardson's distribution.
\end{abstract}

Keywords: Turbulent transport; Relative dispersion

\section{Introduction}

It is known since Taylor's seminal work [1] that tracers transported by a turbulent flow approach a diffuse behavior on time scales much longer than the Lagrangian correlation time of the flow. These ideas are now commonly used in applications, as for instance in air quality control, to model effective mixing properties in terms of an eddy diffusivity. Such models give a good handle on long-term averages and are successfully used to determine, for instance, possible health hazards linked to a long exposure downstream a pollutant source. However they are unable to capture strong local fluctuations stemming from the complex structure of the turbulent flow. Such events cannot be directly predicted from the averaged concentration field as they relate to higher-order moments. Accessing these fluctuations is crucial in order to quantify for instance the likeliness of finding a local concentration exceeding a high threshold.

Second-order statistics, such as the variance of a transported concentration field and more generally the spatial correlation of a passive scalar, are statistically related to the relative motion of tracers (see, e.g., [2]). The problem consists then in

*Corresponding author. Email: jeremie.bec@oca.eu 
investigating the time evolution of the separation $\boldsymbol{R}(t)=\boldsymbol{X}_{1}(t)-\boldsymbol{X}_{2}(t)$ between two Lagrangian trajectories. In turbulence, the distance $|\boldsymbol{R}|$ follows RichardsonObukhov superdiffusive law $\left\langle|\boldsymbol{R}(t)|^{2}\right\rangle \sim \epsilon t^{3}$, where $\epsilon$ is the mean rate of kinetic energy dissipation. The long-term behavior is thus becoming independent of the initial separation $|\boldsymbol{R}(0)|=r_{0}$, whence the designation of explosive pair separation. In his original arguments to derive this law, Richardson [3] assumed that pair separation is a diffusion process with a scale dependent diffusivity $K$. Then the transition probability density $p\left(r, t \mid r_{0}, 0\right)$ that two tracers are at a distance $|\boldsymbol{R}(t)|=r$ knowing that they were initially separated by $|\boldsymbol{R}(0)|=r_{0}$ satisfies the Fokker-Planck equation

$$
\partial_{t} p=\frac{1}{r^{2}} \partial_{r}\left[r^{2} K(r) \partial_{r} p\right]
$$

The atmospheric measurements of Richardson, later refined by Obukhov [4] in view of Kolmogorov 1941 phenomenology, led to assume that for separations $r$ within the inertial range of turbulence $K(r) \propto \epsilon^{1 / 3} r^{4 / 3}$. This implies that, at large times, $\left\langle|\boldsymbol{R}(t)|^{2}\right\rangle \propto \epsilon t^{3}$ and the transition probability reads

$$
p\left(r, t \mid r_{0}, 0\right) \propto \frac{r^{2}}{\left\langle|\boldsymbol{R}(t)|^{2}\right\rangle^{3 / 2}} \exp \left[-\frac{A r^{2 / 3}}{\left\langle|\boldsymbol{R}(t)|^{2}\right\rangle^{1 / 3}}\right]
$$

where $A$ is a positive constant. The considerations leading to the Fokker-Planck equation (11) rely on the use of a central-limit theorem for $\boldsymbol{R}(t)$ and thus on the hypothesis that the velocity difference between the two tracers is correlated over timescales much smaller than those of interest. As noticed for instance in [5], such an hypothesis can hardly be invoked. It is indeed known in turbulence that eddies of size $r$ are correlated over a time of the order of their turnover time $\tau_{r} \sim \epsilon^{-1 / 3} r^{2 / 3}$. Hence for separations that grow like $r \sim \epsilon^{1 / 2} t^{3 / 2}$, one has $\tau_{r} \sim t$, so that the dominant flow structures in the separation dynamics are in principle correlated over timescales of the order of the observation time. Despite such shortcomings, the diffusive approach proposed by Richardson and in particular the explicit form (2) for the transition probability density have proven being relevant in some asymptotics [6 -10]. Also, much work on relative dispersion has used it as a basis. For instance, improvements of (2) were proposed using modified versions of the eddy diffusivity $K(r)$, adding a time dependence [11, 12] or, more recently, including finite Reynolds number effects [13]. All of these improvements strongly alter the functional form of the large- $r$ tail of the transition probability density. Nevertheless, the physical mechanisms leading to the de-correlation of velocity differences and to models based on eddy diffusivity are still unclear and many questions remain open. For instance, the speed of convergence to the Richardson-Obukhov law and the form of subleading terms are still not known.

The first work dealing with the way pair separations converge to a superdiffusive behavior is due to Batchelor [14]. He argued that the explosive $t^{3}$ law is preceded by a ballistic phase during which the tracers keep their initial velocity and separate linearly in time, i.e. $R(t)-r_{0} \simeq t \delta_{r_{0}} u \sim t\left(\epsilon r_{0}\right)^{1 / 3}$, up to a time $\tau_{r_{0}} \sim \epsilon^{-1 / 3} r_{0}^{2 / 3}$, which is equal to the eddy turnover time associated to the initial separation. This first regime where velocity remains strongly correlated can clearly not be described by eddy-diffusivity approaches. Various stochastic models have been designed to catch the two regimes. Most of them are based on the observation that the acceleration difference between the two tracers is shortly correlated and do not assume that velocity differences get uncorrelated. The pair separation and the velocity difference can then be approximated as coupled Markovian diffusive processes (see [15, 16] 
for reviews). The usual path for designing such models requires imposing some constraints on the drift and the diffusion terms. Thomson [17] argued that they should satisfy the well-mixed condition: when averaging over uniformly separated pairs inside the whole inertial range, the statistics of velocity differences between the two tracers has to recover Eulerian two-point statistics. Definitively devising an admissible model requires an input from Eulerian statistics [18]. It is known in turbulence that the distribution of velocity differences is neither self-similar nor Gaussian (see, e.g., [19]). Such models become thus so complicated that they can hardly be used to improve the understanding of the underlying phenomenology and, at the same time, they are not easily amenable for an analytical treatment.

The goal of the present work is to give some new phenomenological understanding of the problem of turbulent relative dispersion. For this, we make use of large-scale direct numerical simulations and essentially focus our study on the role of violent events leading to strong fluctuations. To give grounds to possible improvements of models for relative dispersion, we introduce some new observables, measure their behavior, and interpret the results mostly in terms of geometrical arguments. The paper is organized as follows. We first report in Sec. 2 numerical results on the time evolution of averaged separations and of higher-order statistics; this is an extension of some of our recent work [20]. In Sec. 3 we focus on the behavior of velocity differences and discuss the question of intermittency and of a possible multiscaling for the time evolution of their moments. We also introduce a mixed moment of separation and velocity difference that attains quickly a scaling regime (independent on time and initial separations). In Sec. 4, we apply previous results and make use of geometrical considerations to investigate the very large and small fluctuations of the inter-trajectory distance. Finally, Sec. 5 encompasses concluding remarks and discuss open questions.

\section{Convergence of separation statistics to a scaling regime}

\subsection{Numerical results on the mean-square displacement}

We report in this section numerical results on the time evolution of the averaged squared distance between tracers. The aim is to provide a first insight on the numerical simulations that we have used and on the typical values of the timescales and lengthscales that are used throughout this paper. One of the major difficulties encountered when attacking numerically the problem of turbulent explosive separation is that it requires a huge timescale separation. Indeed, to observe RichardsonObukhov $t^{3}$ superdiffusive regime, one needs to follow particle pairs on a time $t$ much longer than the eddy turnover time $\tau_{r_{0}} \sim \epsilon^{-1 / 3} r_{0}^{2 / 3}$ associated to their initial separation $|\boldsymbol{R}(0)|=r_{0}$ and much smaller than the time $\tau_{L}$ associated to the integral scale $L$. Also, it is in principle required that the initial distance $r_{0}$ belongs to the inertial range, so that its associated turnover time $\tau_{r_{0}}$ has to be much larger than the Kolmogorov dissipative timescale $\tau_{\eta}=(\nu / \epsilon)^{1 / 2}$. We should thus have $\tau_{\eta} \ll \tau_{r_{0}} \ll t \ll \tau_{L}$. To give a concrete idea, if we require that these timescales are separated by at least a decade, this implies that one should have a Taylor-based Reynolds number $R_{\lambda}=\sqrt{15}\left(\tau_{L} / \tau_{\eta}\right) \gtrsim 4000$. Such a high level of turbulence is still far from the current state of direct numerical simulations and also from presentday experimental setups where accurate particle tracking techniques can be used (see [9, 21] for recent reviews). For this reason, there is an important need for understanding the full process of convergence to the $t^{3}$ law in order to predict and detect for instance possible subleading corrections. This is the spirit in which we have tried to present this work. 
Table 1. Parameters of the numerical simulations

\begin{tabular}{ccccccccccc}
\hline$N$ & $R_{\lambda}$ & $\delta x$ & $\delta t$ & $\nu$ & $\epsilon$ & $u_{\mathrm{rms}}$ & $\eta$ & $\tau_{\eta}$ & $L$ & $\tau_{L}$ \\
\hline $2048^{3}$ & 460 & $3.7 \cdot 10^{-3}$ & $6 \cdot 10^{-4}$ & $2.5 \cdot 10^{-5}$ & $3.6 \cdot 10^{-3}$ & 0.19 & $1.4 \cdot 10^{-3}$ & 0.083 & 1.85 & 9.9 \\
\hline $4096^{3}$ & 730 & $1.53 \cdot 10^{-3}$ & $1.2 \cdot 10^{-3}$ & $1.0 \cdot 10^{-5}$ & $3.8 \cdot 10^{-3}$ & 0.19 & $7.2 \cdot 10^{-4}$ & 0.05 & 1.85 & 9.6 \\
\hline
\end{tabular}

$N$ is the number of grid points, $R_{\lambda}$ the Taylor-based Reynolds number, $\delta x$ the grid spacing, $\delta t$ the time step, $\nu$ the kinematic viscosity, $\epsilon$ the averaged energy dissipation rate, $u_{\mathrm{rms}}$ the root-mean square velocity, $\eta=\left(\nu^{3} / \epsilon\right)^{1 / 4}$ the Kolmogorov dissipative scale, $\tau_{\eta}=(\nu / \epsilon)^{1 / 2}$ the associated turnover time, $L=u_{\mathrm{rms}}^{3} / \epsilon$ the integral scale and $\tau_{L}=L / u_{\mathrm{rms}}$ the associated large-scale turnover time.

To investigate such issues, we have performed direct numerical simulations of the incompressible Navier-Stokes equation in the three-dimensional $2 \pi$-periodic domain using a standard pseudo-Fourier-spectral solver with a third-order RungeKutta time marching. Such a method is well adapted to incompressible homogeneous and isotropic turbulence at high Reynolds numbers with an extended inertial range of scales. It has the advantages of combining a high degree of accuracy with very good performances on massive parallel supercomputers such as BlueGene systems and large Intel/AMD clusters. We have used two sets of simulations whose parameters are summarized in table 1 (more details on these simulations can be found in [22]). To maintain a statistical steady state, the flow is forced by keeping constant the energy content of the two first shells of wavenumbers in Fourier space.
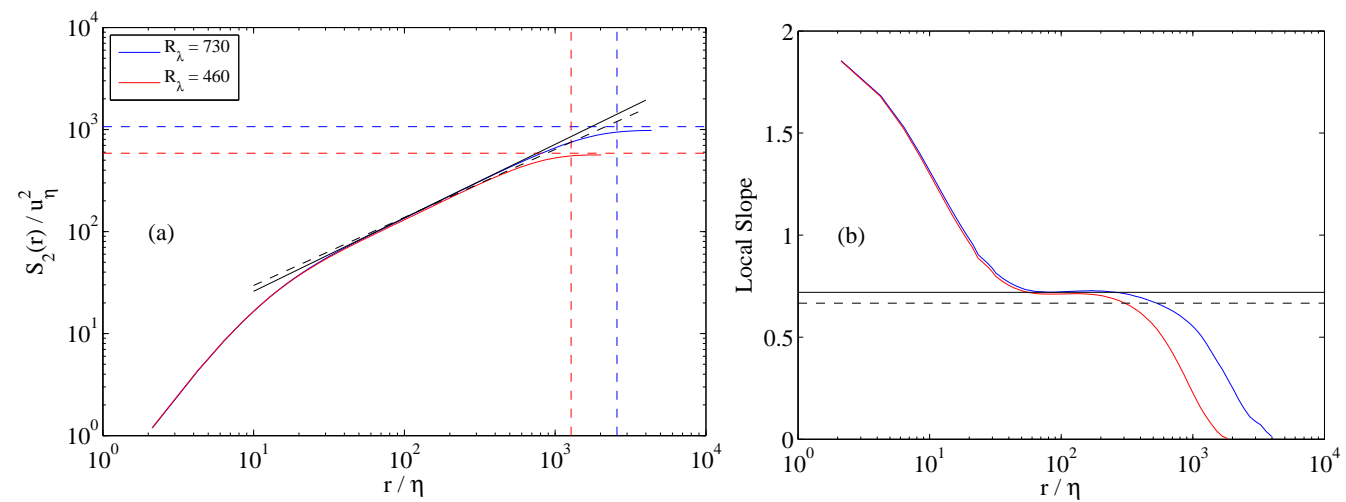

Figure 1. (a) Second-order Eulerian structure function $S_{2}(r)=\left\langle|\boldsymbol{u}(\boldsymbol{x}+\boldsymbol{r}, t)-\boldsymbol{u}(\boldsymbol{x}, t)|^{2}\right\rangle$ for the two values of the Reynolds number investigated in this paper. The solid line represents Kolmogorov 1941 scaling $S_{2}(r) \simeq(11 / 3) C_{2}(\epsilon r)^{2 / 3}$ with $C_{2}=2.13$. The dashed line corresponds to She-Lévêque anomalous scaling with $\zeta_{2} \approx 0.696$. The vertical and horizontal colored dashed lines indicate the integral scale and the largescale velocity, respectively. (b) Logarithmic derivative $\mathrm{d} \log S_{2} / \mathrm{d} \log r$. The two horizontal lines encode the two scalings shown in Fig. (a).

The Eulerian second-order structure function measured from these two simulations are represented in Fig. 1. As seen there, the largest one develops a significant scaling range where deviations from Kolmogorov 1941 scaling start to be visible.

For each value of the Reynolds number, the flow is seeded with $10^{7}$ tracer particles whose motion is integrated using tri-linear interpolation. After a time sufficiently long to have converged to a statistical steady state, we start the analysis of the dispersion of tracer pairs. For this, we label at a fixed initial time (that we fix here to be $t=0$ ) all couples whose distance $|\boldsymbol{R}(0)|=\left|\boldsymbol{X}_{1}(0)-\boldsymbol{X}_{2}(0)\right|$ is equal to $r_{0} \pm \eta$ for $r_{0} \leq 16 \eta$ and equal to $r_{0}(1 \pm 2 \%)$ for $r_{0}>16 \eta$ with $r_{0}=2,3,4,6,8,12,16,24,32,48,64,96,128$, and $192 \eta$. This bining was chosen such that each family indexed by $r_{0}$ contains a few hundreds of thousands of pairs. We then track forward in time all indexed pairs and perform statistics conditioned on their initial separation $r_{0}$. For the sake of simplicity we denote by $\langle\cdot\rangle$ the Lagrangian ensemble average conditioned on $r_{0}$. 

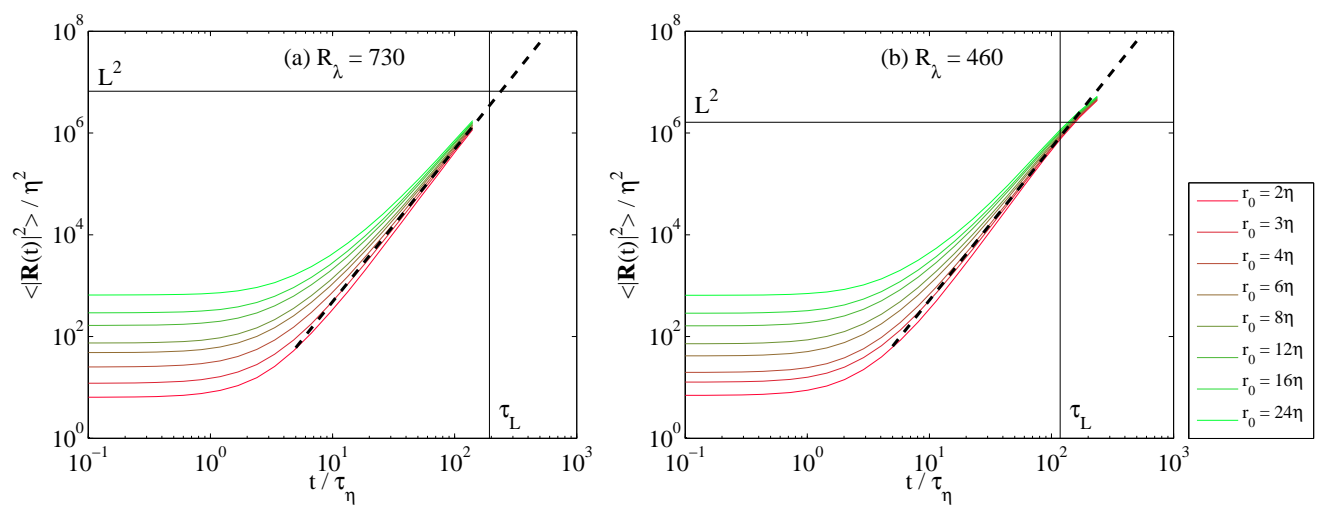

Figure 2. Time-evolution of the mean-squared distance for $R_{\lambda}=730$ (a) and $R_{\lambda}=460$ (b) for various initial separations $r_{0}$ as labeled. The horizontal and vertical solid lines represent the integral scale $L$ and its associated turnover time $\tau_{L}$, respectively. The dashed line corresponds to the explosive RichardsonObukhov law (3) with $g=0.52$.
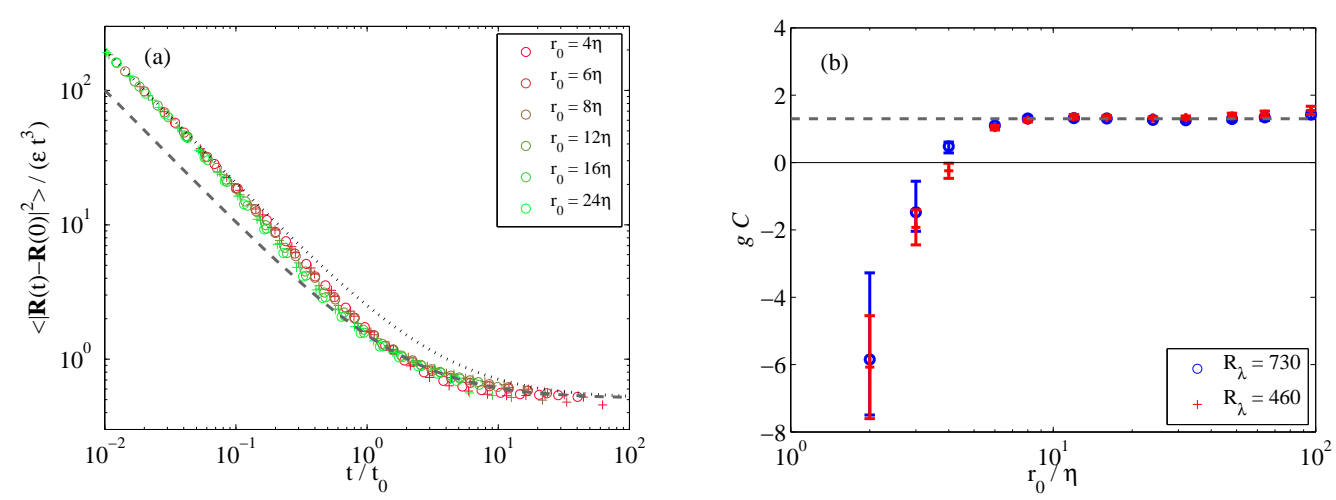

Figure 3. (a) Compensated mean-squared displacement $\left\langle|\boldsymbol{R}(t)-\boldsymbol{R}(0)|^{2}\right\rangle /\left(\epsilon t^{3}\right)$ as a function of $t / t_{0}$ with $t_{0}=S_{2}\left(r_{0}\right) /(2 \epsilon)$ for various initial separations and $R_{\lambda}=730(\mathrm{o})$ and $R_{\lambda}=460(+)$. The two curves show behaviors of the form $\left\langle|\boldsymbol{R}(t)-\boldsymbol{R}(0)|^{2}\right\rangle \simeq g \epsilon t^{3}+A t^{2}$, with $A=S_{2}\left(r_{0}\right)$, given by Batchelor's ballistic regime (black dotted line), and $A=2.5 / t_{0}^{2}$ (grey dashed line). (b) Measured value of the constant $g C$ in front of the subleading term as a function of the initial separation. It stabilizes to $C \approx 1.3 / g \approx 2.5$ for $r_{0} \gg \eta$; this value is represented as a dashed line.

Figure 2 shows for the two simulations the time evolution of the mean squared distance $\left\langle|\boldsymbol{R}(t)|^{2}\right\rangle$ for various values of the initial separation $r_{0}$. Times and space are there represented in dissipative-scale units. After a transient (which roughly corresponds to Batchelor's ballistic regime), the mean-squared distance approaches the explosive Richardson-Obukhov regime

$$
\left\langle|\boldsymbol{R}(t)|^{2}\right\rangle \simeq g \epsilon t^{3}
$$

We observe for both values of the Reynolds number a Richardson-Obukhov constant $g \approx 0.52 \pm 0.05$. The low accuracy with which this constant is determined comes from the fact that, even at the higher resolution, the $t^{3}$ scaling is observed in a rather limited time range. This is even clearer from Fig. 3 (a), which shows the compensated mean squared increase of the distance $\left\langle|\boldsymbol{R}(t)-\boldsymbol{R}(0)|^{2}\right\rangle /\left(\epsilon t^{3}\right)$. On this figure, the time has been rescaled by $t_{0}=S_{2}\left(r_{0}\right) /(2 \epsilon)$, where $S_{2}$ designates the second-order Eulerian structure function with absolute values. The choice of such a timescale was motivated in [20] as that of deviations from Batchelor's initial ballistic regime. Surprisingly, the data shown in Fig. 3 (a) corresponding to various initial separations $r_{0}$ far enough in the inertial range seem to collapse. This suggests that the timescale $t_{0}$ contains most of the dependence of pair dispersion upon the initial separation $r_{0}$. Also data indicate that the subdominant terms in 
(3) are $\propto t^{2}$, leading to postulate $\left\langle|\boldsymbol{R}(t)|^{2}\right\rangle \simeq g \epsilon t^{3}\left(1+C t_{0} / t\right)$, with a constant $C$ independent of $r_{0}$ when $r_{0} \gg \eta$. The product of the constants $g$ and $C$ has been estimated numerically and results are shown in Fig. 3 (b). One can clearly see that $C \approx 1.3 / g \approx 2.5$ when $r_{0} \gtrsim 8 \eta$.

One can also see from the figure that $C<0$ for $r_{0} \lesssim 4 \eta$. Thus, for dissipativerange initial separations, the asymptotic $t^{3}$ behavior is attained from below. This can lead for such values of $r_{0}$ to an intermediate time range where the mean squared distance grows even faster than the explosive $t^{3}$ law, as for instance observed in [7]. Another remark that can be drawn from the data is that, independently of the Reynolds number, the constant $C$ is equal to zero for $r_{0} \approx 4 \eta$. The first subleading terms are then $\propto t$, so that the convergence to the $t^{3}$ law is much faster for such an initial separation than for others. This observation could be useful for experimentalists to optimize their setup. However, such small values of $r_{0}$ are clearly not representative of the inertial-range behavior.

\subsection{Higher-order statistics}
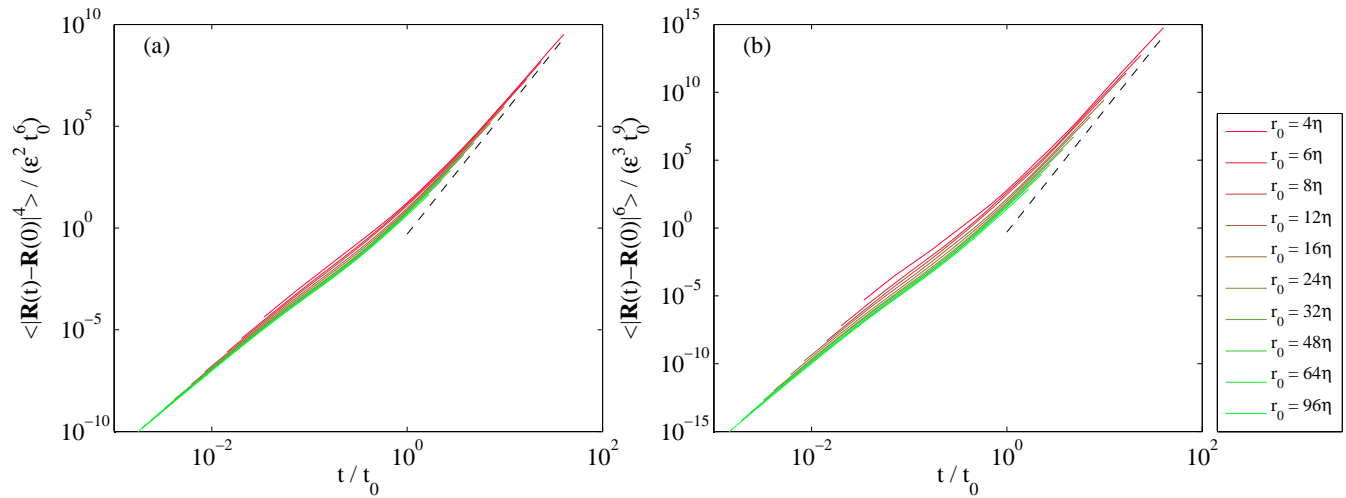

Figure 4. (a) Fourth-order moment $\left\langle|\boldsymbol{R}(t)-\boldsymbol{R}(0)|^{4}\right\rangle$ and (b) sixth-order moment $\left\langle|\boldsymbol{R}(t)-\boldsymbol{R}(0)|^{6}\right\rangle$ as function of $t / t_{0}$ for $R_{\lambda}=730$. Both curves are normalized such that their expected long-time behavior is $\propto\left(t / t_{0}\right)^{6}$ and $\propto\left(t / t_{0}\right)^{9}$, respectively. The black dashed lines represent such behaviors.

We now turn to investigating the large-time behavior of higher-order moments of the separation. Figure 4 shows the time evolution of $\left\langle|\boldsymbol{R}(t)-\boldsymbol{R}(0)|^{4}\right\rangle$ (a) and of $\left\langle|\boldsymbol{R}(t)-\boldsymbol{R}(0)|^{6}\right\rangle$ (b). At times smaller than $t_{0}$ the separation grows ballistically, so that $\left\langle|\boldsymbol{R}(t)-\boldsymbol{R}(0)|^{p}\right\rangle \simeq t^{p}\left\langle|\boldsymbol{V}(0)|^{p}\right\rangle$ where $\boldsymbol{V}(t)=\boldsymbol{u}\left(\boldsymbol{X}_{1}, t\right)-\boldsymbol{u}\left(\boldsymbol{X}_{1}, t\right)$ denotes the velocity difference between the two tracers. The fact that we have chosen to rescale time by $t_{0}$ (which depends on second-order statistics of the initial velocity difference) implies that the moments do not collapse in this regime because of Eulerian multiscaling. However the collapse occurs for $t \gg t_{0}$ where these two moments grow like $t^{6}$ and $t^{9}$, respectively, with possible minute deviations. The measured power-laws give evidence that, at sufficiently long times, inter-tracer distances follow a scale-invariant law. Also the observed collapses indicate that $t_{0}$ could be again the time of convergence to such a behavior.

The presence of a scale-invariant regime is also clear when making use of ideas borrowed from extended self-similarity and representing these two moments as a function of $\left\langle|\boldsymbol{R}(t)-\boldsymbol{R}(0)|^{2}\right\rangle$ (see Fig. 5). This time, for a fixed $r_{0}$, the smallest separations correspond to the ballistic regime. There, we trivially have $\left\langle|\boldsymbol{R}(t)-\boldsymbol{R}(0)|^{p}\right\rangle /\left\langle|\boldsymbol{R}(t)-\boldsymbol{R}(0)|^{2}\right\rangle^{p / 2} \simeq\left\langle|\boldsymbol{V}(0)|^{p}\right\rangle /\left\langle|\boldsymbol{V}(0)|^{2}\right\rangle^{p / 2}$, which has a weak dependence on $r_{0}$, because of an intermittent distribution of Eulerian velocity increments, but does not depend on time. This normal scaling can be observed for 

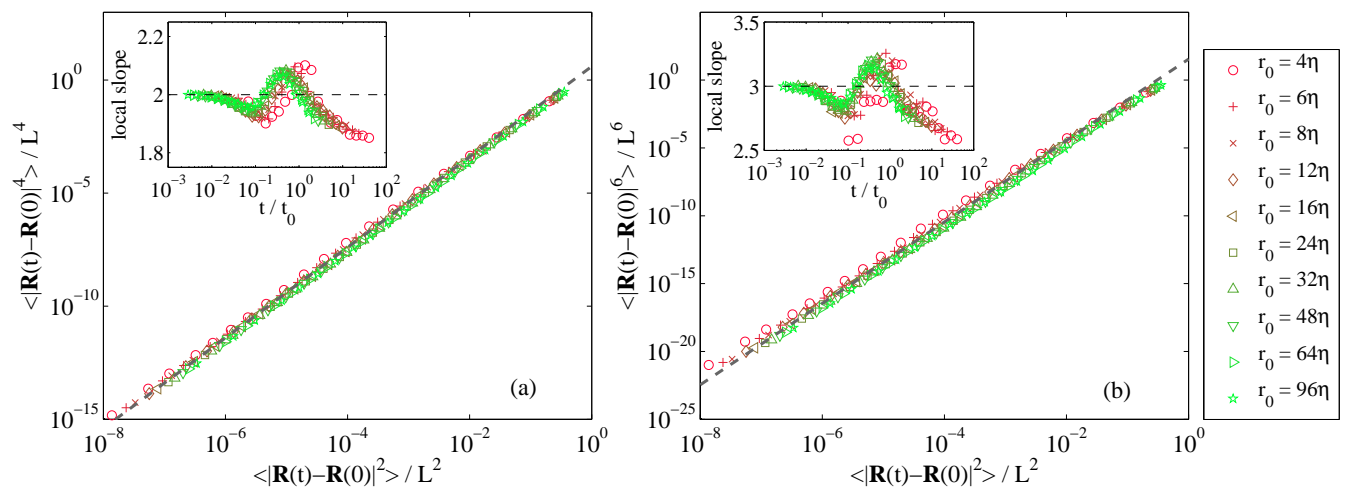

Figure 5. Fourth (a) and sixth (b) order moments of $|\boldsymbol{R}(t)-\boldsymbol{R}(0)|$ as a function of its second-order moment for $R_{\lambda}=730$. The two gray dashed lines show a scale-invariant behavior, i.e. $\left\langle|\boldsymbol{R}(t)-\boldsymbol{R}(0)|^{4}\right\rangle \propto$ $\left\langle|\boldsymbol{R}(t)-\boldsymbol{R}(0)|^{2}\right\rangle^{2}$ and $\left\langle|\boldsymbol{R}(t)-\boldsymbol{R}(0)|^{6}\right\rangle \propto\left\langle|\boldsymbol{R}(t)-\boldsymbol{R}(0)|^{2}\right\rangle^{3}$, respectively. The two insets show the associated local slopes, that is the logarithmic derivatives $\mathrm{d} \log \left\langle|\boldsymbol{R}(t)-\boldsymbol{R}(0)|^{p}\right\rangle / \mathrm{d} \log \left\langle|\boldsymbol{R}(t)-\boldsymbol{R}(0)|^{2}\right\rangle$, together with the normal scalings represented as dashed lines.

$t \ll t_{0}$ in the insets of Fig. 5, which represent the logarithmic derivatives of the high-order moments with respect to the second order. At times of the order of $t_{0}$, noticeable deviations to normal scaling can be observed. Finally, at much larger scales, data corresponding to different values of the initial separation $r_{0}$ collapse but the curves start to bend down. One observes in the insets that the associated local slopes approach values clearly smaller than those corresponding to normal scaling. This gives evidence of a rather weak intermittency in the distribution of tracer separations. Note that the presented measurements were performed for $R_{\lambda}=730$ but the same behavior has been observed for $R_{\lambda}=460$.

To our knowledge, the most convincing observation of an intermittent behavior in pair dispersion has been based on an exit-time analysis [23]. However, the relation of such fixed-scale statistics to the usual fixed-time measurements we report here requires to consider pair separation velocities. As we will see in next Section, the velocity difference between two tracers displays statistics that are much more intermittent than those for pair separation. This implies that there is no contradiction between an almost normal scaling for distances as a function of time and an anomalous behavior of exit times as a function of distance.
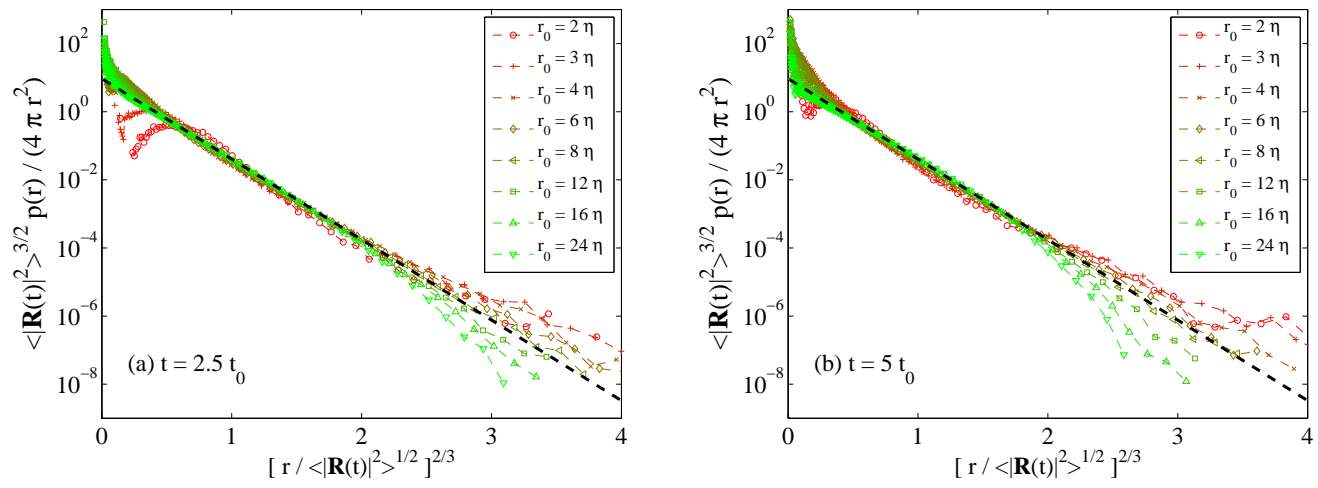

Figure 6. Probability density function of the distance $r$ at time $t=2.5 t_{0}$ (a) and $t=5 t_{0}$ (b) and for various values of the initial separation. We have here normalized it by $4 \pi r^{2}$ and represented on a $\log y$ axis as a function of $r /\left\langle|\boldsymbol{R}(t)|^{2}\right\rangle^{1 / 2}$. With such a choice, Richardson's diffusive density distribution (2) appears as a straight line (represented here as a black dashed line).

To investigate further this weak intermittency in the separation distribution, we have represented in Fig. 6 the probability density function (PDF) of the distance $|\boldsymbol{R}(t)|$ for various initial separation and at times where we expect to have almost 
converged to the explosive regime, namely at $t=2.5 t_{0}$ (a) and $t=5 t_{0}$ (b). Such measurements are compared to Richardson's diffusive law (2). Data suggest that a large part of the PDF core (for $0.4 \lesssim|\boldsymbol{R}(t)| /\left\langle|\boldsymbol{R}(t)|^{2}\right\rangle^{1 / 2} \lesssim 4$ at time $t=5 t_{0}$ ) is very well described by Richardson's approach. However, deviations are observed in the far tails, at both small and large values of the separation. Such an observation is consistent with previous numerical observations [7, 13, 23]. Apparently, these deviations affect only weakly the moments we have considered above. We will come back to investigating and characterizing them in Sec. 4.

\section{Statistics of velocity differences}

\subsection{A diffusive behavior?}

In this section, we are interested in the behavior of the velocity difference $\boldsymbol{V}(t)=$ $\boldsymbol{u}\left(\boldsymbol{X}_{1}(t), t\right)-\boldsymbol{u}\left(\boldsymbol{X}_{2}(t), t\right)$ between two tracers as a function of time. Initially, the statistics of $\boldsymbol{V}(0)$ are exactly given by the Eulerian statistics of velocity increments at a separation $r_{0}$. At large times, an explosive $t^{3}$ behavior for distances implies that $\left\langle|\boldsymbol{V}(t)|^{2}\right\rangle \propto \epsilon t$. A naive picture would consist in interpolating between these two behaviors by assuming that

$$
\left\langle|\boldsymbol{V}(t)|^{2}\right\rangle \simeq S_{2}\left(r_{0}\right)+h \epsilon t,
$$

where $h$ is a positive constant that cannot be straightforwardly expressed as a function of $g$. As argued in [20] one of the two terms, which correspond to the ballistic or to the Richardson-Obukhov regime, is dominant when $t$ is much smaller or much longer than $t_{0}=S_{2}\left(r_{0}\right) /(2 \epsilon)$. As seen from Fig. 7 (a) such a form with $h=2.4$ (represented as a dashed line) seems to be a good first-order approximation. However, this picture is not completely satisfactory. When zooming closer to the initial times, one observes that the averaged pair kinetic energy $\left\langle|\boldsymbol{V}(t)|^{2}\right\rangle$ first starts by decreasing for a time of the order of a few tens of $t_{0}$. We indeed know that initially

$$
\boldsymbol{V}(t)=\boldsymbol{V}(0)+t \boldsymbol{A}(0)+\mathcal{O}\left(t^{2}\right)
$$
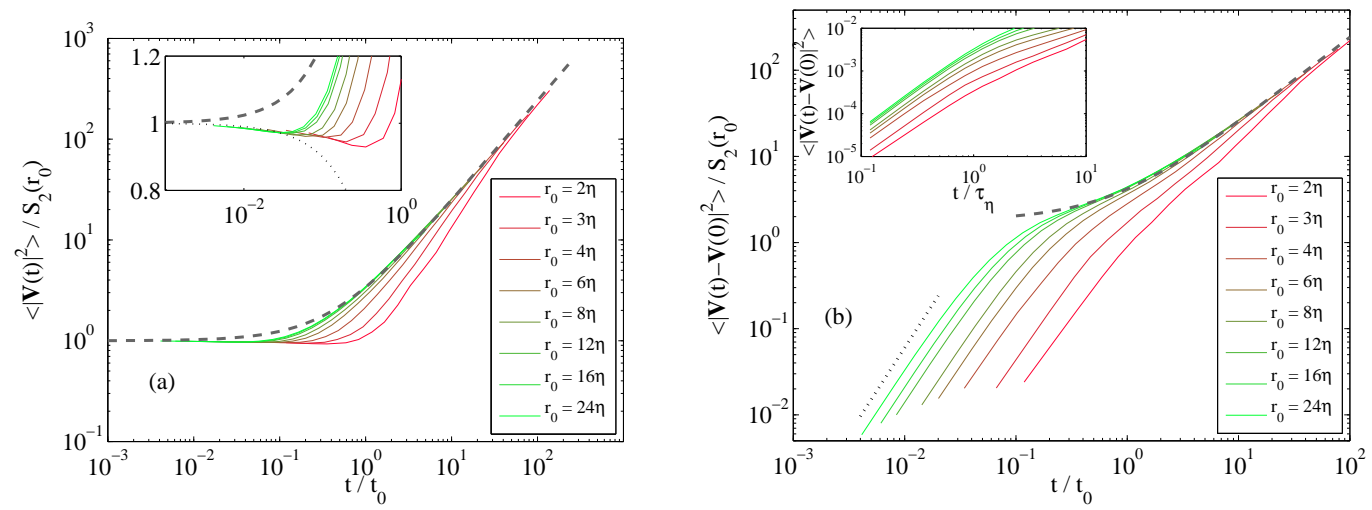

Figure 7. Time behavior of the mean-squared velocity difference for $R_{\lambda}=730$. (a) $\left\langle|\boldsymbol{V}(t)|^{2}\right\rangle$, normalized by its initial value, as a function of $t / t_{0}$; the gray dashed line is a behavior of the form $\left\langle|\boldsymbol{V}(t)|^{2}\right\rangle \simeq$ $S_{2}\left(r_{0}\right)+h \epsilon t$ with $h=2.4$. Inset: same but zoomed at short times; the black dotted line is the initial decrease associated to kinetic energy dissipation: $\left\langle|\boldsymbol{V}(t)|^{2}\right\rangle \simeq S_{2}\left(r_{0}\right)-2 \epsilon t$. (b) Time behavior of the velocity change $\left\langle|\boldsymbol{V}(t)-\boldsymbol{V}(0)|^{2}\right\rangle$; the dashed curve is $\left\langle|\boldsymbol{V}(t)-\boldsymbol{V}(0)|^{2}\right\rangle \simeq S_{2}\left(r_{0}\right)+h \epsilon t$; the dotted line has slope 2 and corresponds to the initial abrupt variation of the velocity difference. Inset: same but rescaling time by $\tau_{\eta}$. 
where $\boldsymbol{A}(0)$ denotes the initial difference of acceleration between the two tracers. This approximation leads to $\left\langle|\boldsymbol{V}(t)|^{2}\right\rangle \simeq S_{2}\left(r_{0}\right)-2 \epsilon t$ (see, e.g., [20]). According to our numerical data, this regime seems to reproduce well what is happening for times up to $\approx 0.01 t_{0}$ - see inset of Fig. 7 (a). The fact that this prediction ceases to describe the data after such a short timescale (that is significantly smaller than $t_{0}$ instead of being of its order) indicates that the subleading terms in (5) then become important. As a consequence, depending on the quantity we are interested in, Batchelor's ballistic regime might end very quickly.

After this subtle initial decrease, the averaged squared velocity difference amplitude tends asymptotically to a behavior $\propto t$. However this process occurs in a noticeably different manner to that of convergence of squared separation to Richardson $t^{3}$ law: irrespective of the initial separation $r_{0}$, the quantity $\left\langle|\boldsymbol{V}(t)|^{2}\right\rangle$ always approaches from below the asymptotic behavior. In other terms, we observe that for a fixed value of $t / t_{0}$, the pair kinetic energy $\left\langle|\boldsymbol{V}(t)|^{2}\right\rangle$ is an increasing function of $r_{0} / \eta$ and seems to converge to (44) only in the limit $r_{0} / \eta \rightarrow \infty$.

At large times, the mean-squared velocity difference grows linearly. This behavior, together with the fact that acceleration differences are correlated over timescales of the order of the Kolmogorov dissipative time $\tau_{\eta}$, suggest that velocity differences have a diffusive behavior for $t \gg \tau_{\eta}$ (see [20]). If this was exact, it would lead to the behavior (4) as the temporal increments of $\boldsymbol{V}$ would be independent of its initial value. Also, a purely diffusive behavior of $\boldsymbol{V}$ would result in the fact that its mean-squared temporal increment $\left\langle|\boldsymbol{V}(t)-\boldsymbol{V}(0)|^{2}\right\rangle$ is $\propto t$ for all times $t \gg \tau_{\eta}$. As seen in Fig. 7 (b), the diffusive regime is actually only asymptotic. Also, data show that $\boldsymbol{V}(t)$ initially changes on times of the order of $\tau_{\eta}$ by a factor of the order of its typical initial value $\left[S_{2}\left(r_{0}\right)\right]^{1 / 2}$. This abrupt evolution can be interpreted phenomenologically. With some finite probability, one of the two tracers is within a vortex filament at time $t_{0}$. The typical energy content of this filament will contribute to the value of $S_{2}\left(r_{0}\right)$. However, after a time $t$ of the order of $\tau_{\eta}$, the trajectory of this tracer will have turned around this filament, so that its velocity will have completely changed orientation (without changing much its amplitude). This will result in $|\boldsymbol{V}(t)-\boldsymbol{V}(0)| \sim|\boldsymbol{V}(t)|$, explaining the observed behavior. As a result of this sudden kinematic variations of velocity differences, $\left\langle|\boldsymbol{V}(t)-\boldsymbol{V}(0)|^{2}\right\rangle$ behaves in a very similar manner to $\left\langle|\boldsymbol{V}(t)|^{2}\right\rangle$ for times $t \gg \tau_{\eta}$ (compare the dashed lines in Fig. 7(a) and (b)). Let us also notice that the convergence to this behavior is again from below, irrespective of the initial separation $r_{0}$.

There is hence an abrupt change (occurring on timescales of the order of $\tau_{\eta}$ ) that prevents from determining an effective initial velocity difference and thus from observing a clear diffusive behavior of $\boldsymbol{V}(t)$. However, data suggest that the timescale of convergence to this behavior is, as for separations, of the order of $t_{0}$. To understand further this question, we next turn to investigating the behavior of the longitudinal velocity difference between the tracers.

\subsection{Geometry of longitudinal velocities}

We are here interested in the evolution of the longitudinal component $V^{\|}(t)=$ $\boldsymbol{R}(t) \cdot \boldsymbol{V}(t) /|\boldsymbol{R}(t)|$ of the velocity difference as a function of time. This quantity is important to characterize pair separation as $\mathrm{d}|\boldsymbol{R}| / \mathrm{d} t=V^{\|}$. Initially, the averaged longitudinal velocity vanishes, i.e. $\left\langle V^{\|}(0)\right\rangle=0$; this is due to the statistical stationarity of the fluid flow. For times $t \ll t_{0}$ in the Batchelor's ballistic regime, the pairs are keeping their initial velocity difference and one can easily check that

$$
\left\langle V^{\|}(t)\right\rangle \simeq t\left\langle\left|\boldsymbol{V}^{\perp}(t)\right|^{2}\right\rangle / r_{0}
$$



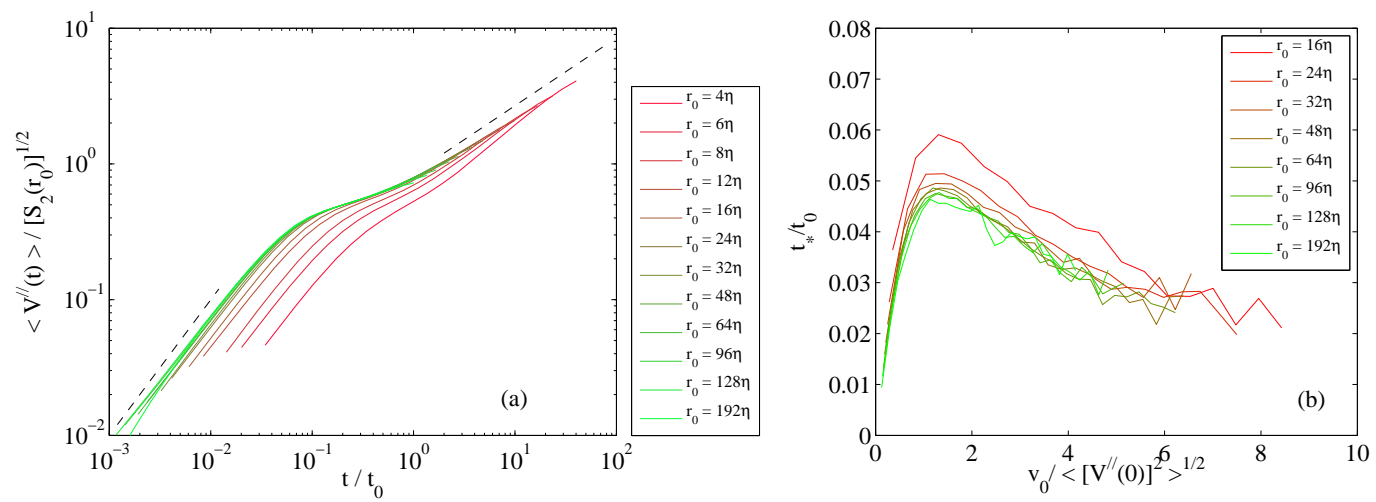

Figure 8. (a) Time evolution of the averaged longitudinal velocity difference $\left\langle V^{\|}(t)\right\rangle$ for various initial separations, as labeled, and for $R_{\lambda}=730$. (b) Averaged time $t_{*}=-r_{0} v_{0} /\left\langle|\boldsymbol{V}(0)|^{2} \mid V^{\|}(0)=v_{0}\right\rangle$ when trajectories with a given initial longitudinal velocity difference $v_{0}$ reach a minimal distance.

where $\boldsymbol{V}^{\perp}$ denotes the components of $\boldsymbol{V}$ that are transverse to $\boldsymbol{R}$. It is thus clear that the average velocity at which tracer trajectories separate immediately becomes positive. Figure 8 (a), which represents the time evolution of $\left\langle V^{\|}(t)\right\rangle$, shows without doubt this initial linear growth. This increase of the longitudinal velocity difference has an interesting geometrical interpretation. If all pairs were to maintain indefinitely their initial velocity differences $\boldsymbol{V}(0)$, it is clear that they would eventually all separate at large times. Indeed, they will reach a minimal distance at a finite time $t_{*}=-[\boldsymbol{R}(0) \cdot \boldsymbol{V}(0)] /|\boldsymbol{V}(0)|^{2}=-r_{0} V^{\|}(0) /|\boldsymbol{V}(0)|^{2}$, which is positive for particles that are initially approaching. After the time $t_{*}$, the distance between particles increases and $V^{\|}$becomes positive. This leads to an increase of $\left\langle V^{\|}\right\rangle$that comes from kinematic considerations and is obviously not due to any dynamics imposed by the turbulent flow. To estimate the typical value of the minimal distance at time $t_{*}$, we have performed statistics on pair separation conditioned not only on the initial distance $r_{0}$, but also on the initial longitudinal velocity difference by binning pairs with $V^{\|}(0)=v_{0} \pm \delta v_{0}$. From these statistics we have defined the averaged time $t_{*}=-r_{0} v_{0} /\left\langle|\boldsymbol{V}(0)|^{2} \mid v_{0}\right\rangle$ at which trajectories with a given initial longitudinal velocity difference $v_{0}$ are at a minimal distance. The data are shown in Fig. 8 (b) for various values of $r_{0}$ and as a function of $v_{0}$. We observe that for $r_{0} \gg \eta$ this time approximatively takes the form $t_{*} \simeq t_{0} f\left(v_{0} /\left\langle|\boldsymbol{V}(0)|^{2}\right\rangle^{1 / 2}\right)$, where the function $f(x)$ attains its maximum (roughly equal to 0.05 ) at $x \approx 1$. This confirms the observation made in Fig. 8 (a) that the initial growth of $\left\langle V^{\|}\right\rangle$occurs on a time length of the order of a few hundredths of $t_{0}$.

In addition to the change in the mean longitudinal velocity difference discussed above, numerical measurements show that the full distribution of $V^{\|}$looses its symmetry and develops fatter tails when time increases. Figures 9 (a) and (b) represent the skewness $\mathcal{S}$ and flatness $\mathcal{F}$ of $V^{\|}$as a function of time and for the same initial separations as in Fig. 8 (a). These observables are frequently used in turbulence to quantify the shape of the velocity increment distribution. For twoparticle Lagrangian statistics, they are defined as

$$
\mathcal{S}(t)=\frac{\left\langle\left[V^{\|}(t)-\left\langle V^{\|}(t)\right\rangle\right]^{3}\right\rangle}{\left\langle\left[V^{\|}(t)-\left\langle V^{\|}(t)\right\rangle\right]^{2}\right\rangle^{3 / 2}} \quad \text { and } \quad \mathcal{F}(t)=\frac{\left\langle\left[V^{\|}(t)-\left\langle V^{\|}(t)\right\rangle\right]^{4}\right\rangle}{\left\langle\left[V^{\|}(t)-\left\langle V^{\|}(t)\right\rangle\right]^{2}\right\rangle^{2}} .
$$

As already observed for instance in [24], these quantities strongly vary as a function of time and maintain a marked dependence upon the initial separation $r_{0}$ for rather long times. Figure 9 (a) shows that the skewness of $V^{\|}(t)$ starts from negative values (to be in agreement with the Eulerian $4 / 5$ law) and becomes positive at 

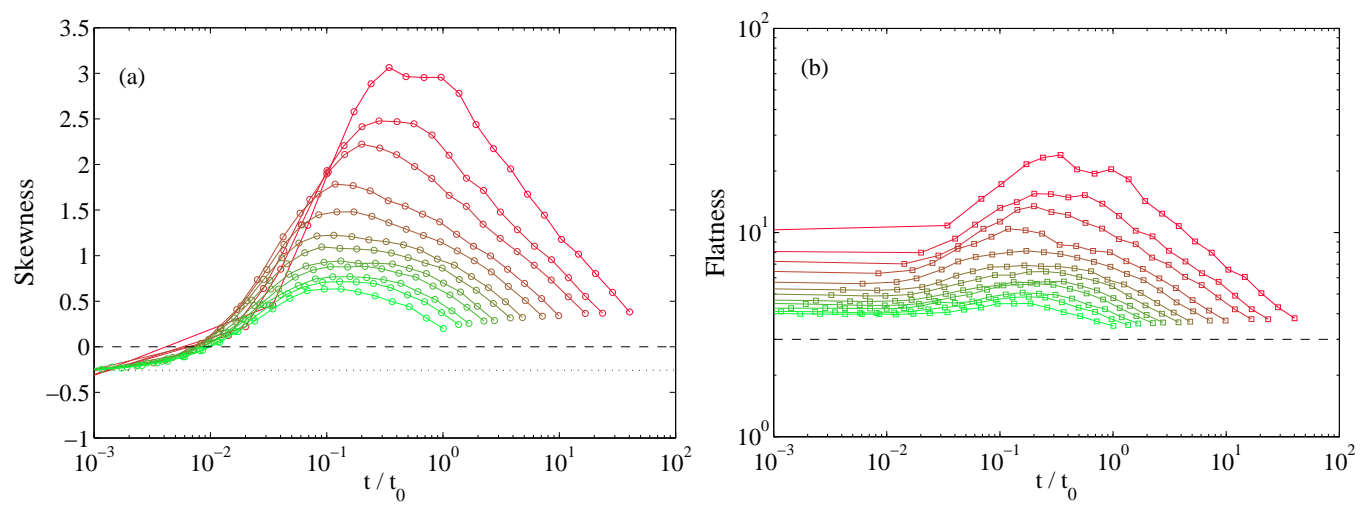

Figure 9. Time evolution of (a) the skewness $\mathcal{S}(t)$ and (b) the flatness $\mathcal{F}(t)$ defined in (7) for the same initial separations $r_{0}$ as in Fig. 8 (a). The dashed lines are the values corresponding to a Gaussian distribution: $\mathcal{S}=0$ and $\mathcal{F}=3$. The dotted line in (a) is the initial value of the skewness obtained when assuming Kolmogorov 1941 scaling, namely $\mathcal{S}=(4 / 5) / C_{2}^{3 / 2}$.

times larger than $\approx 0.01 t_{0}$. This initial change of sign can also be interpreted geometrically in terms of the time $t_{*}$ when initially ballistically approaching pairs begin to move away. However, after this, the curves separate and each of them attains a maximum at times of the order of $t_{0}$ or slightly smaller. This maximal value of the skewness strongly depends on the initial separation: less is $r_{0}$, higher it is. After this maximum, the skewness decreases without attaining an asymptotic regime that would be independent of $r_{0}$. This could be either due to the fact that there is a persistent memory of $r_{0}$ in such quantities or to a contamination by finite Reynolds number (and finite size) effects. The same kind of behavior is observed for the flatness $\mathcal{F}$ of the distribution of $V^{\|}$as seen in Fig. 9 (b). However, the increase of its maximal value when decreasing $r_{0}$ is even more pronounced. Another noticeable difference is that the initial value of $\mathcal{F}$ itself depends on $r_{0}$ and relates to the scale dependence of the Eulerian flatness.
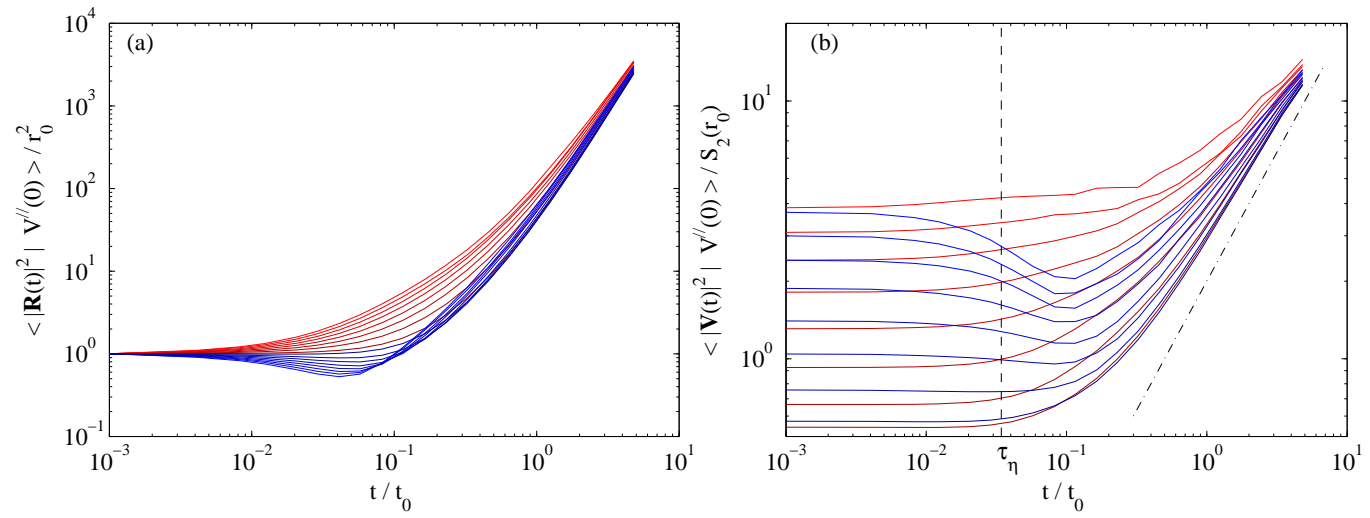

Figure 10. Time evolution for $r_{0}=24 \eta$ of the averaged distance (a) and of the squared velocity difference (b) conditioned on $V^{\|}(0)=v_{0}$ with $\left|v_{0}\right| /\left\langle\left[V^{\|}(0)\right]^{2}\right\rangle^{1 / 2}=0.1,0.4,0.8,1.2, \ldots, 2.8$. The red curves correspond to positive values of $v_{0}$ and the blue ones to negative values. In (b) the dashed-dotted line is $\propto t$

The strong dependence on $r_{0}$ of the skewness and of the flatness can be interpreted phenomenologically in terms of the intermittent nature of velocity increments. A large positive value of $\mathcal{S}$ corresponds to a large probability of having pairs separating faster than the average. Such events will also be responsible for a large value of the flatness $\mathcal{F}$. It is clear that those particles that separate the faster are typically those which are the most separated. Also, in a turbulent flow, the typical value of the velocity increases with scale, so that the particles which get quickly separated are likely to continue separating faster than the average. This is 
evidenced in Fig. 10, which represents the mean squared distance and the averaged squared amplitude of the velocity difference conditioned on the initial value of the longitudinal velocity difference for $r_{0}=24 \eta$. One observes that there is up to the latest time (of the order of $5 t_{0}$ ), a noticeable memory on the initial value of $V^{\|}$. Besides this consideration, one also remarks in Fig. 10 (b) that the pairs having an initially large negative longitudinal velocity difference (the blue curves) dissipate much more kinetic energy than the others. However, this does not prevent them from separating at large times faster than the pairs having initially a smaller velocity difference. We now turn back to the explanation of the long-term dependence on $r_{0}$ of the skewness and flatness of $V^{\|}$. Recall that, in a turbulent flow, violent velocity differences are more probable at small scales than at larger scales. This implies that pairs with a small initial separation are more likely to experience a large (positive or negative) initial velocity difference. This will make them separate faster than the average and thus experience even larger values of the velocity. The rapid and strong increase of fluctuations in their velocity differences is thus due to a kind of snowball effect. We will come back later to quantifying with more accuracy this phenomenon.

\subsection{Intermittency}

All the considerations on the dependence of $V^{\|}$upon the initial separation are also visible in the probability distribution of the longitudinal velocity. Figure 11 (a) shows the centered PDF of $V^{\|}$normalized to unit variance for various times and $r_{0}=12 \eta$. The data clearly show that at times later than $0.01 t_{0}$, there is a change in the sign of the skewness. Also, one sees that the time dependence of the skewness and of the flatness comes from the right tails associated to large velocity differences, supporting the arguments discussed above. The left tails, which corresponds to approaching pairs, seem on the contrary to collapse. The right tail has a very rich behavior. It starts with broadening at times $t<t_{0}$, in agreement with the increase of flatness. For $t>t_{0}$, it then decreases and possibly goes back asymptotically to its initial form. If this was true, it would imply that the distribution of velocity differences keep in memory the initial separation at any later time.
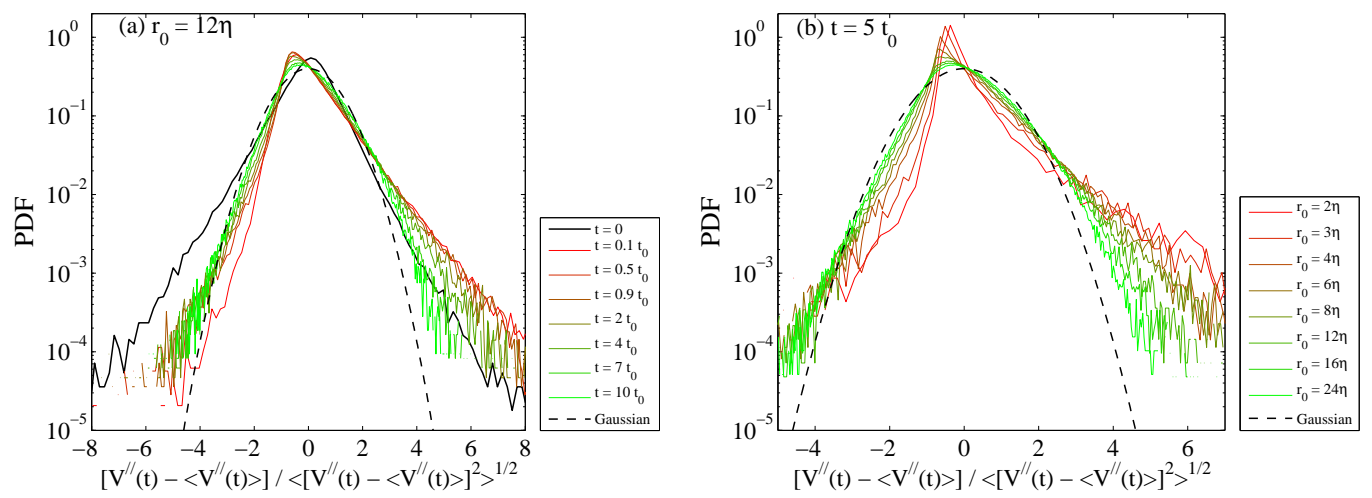

Figure 11. Centered and normalized probability density functions of the longitudinal velocity difference: (a) for $r_{0}=12 \eta$ and various times and (b) for various initial separations and $t=5 t_{0}$. In both cases, the black dashed line shows a Gaussian distribution.

This is also evidenced from Fig. 11(b), which shows the same PDFs for various separations and a time $t=5 t_{0}$ fixed. Again we observe a rather good collapse of the tails associated to negative longitudinal velocity differences, but the right tails display very strong dependence on the initial separation. Clearly, the behavior of this tail is a stretched exponential for $r_{0} \lesssim 8 \eta$ and is faster than exponential for 

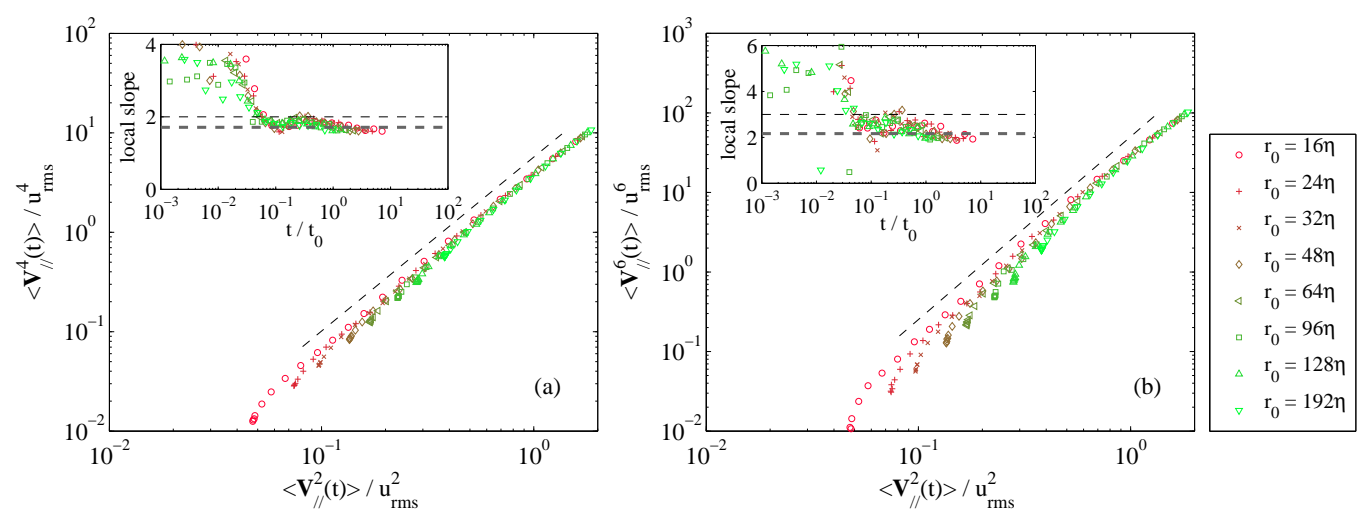

Figure 12. Fourth-order (a) and sixth-order (b) moments of the longitudinal velocity difference as a function of its second-order moment for various times and initial separations. The two dashed lines correspond to a scaling compatible with that of Lagrangian structure functions proposed in [25], namely $\zeta_{4}^{\mathrm{L}} / \zeta_{2}^{\mathrm{L}}=1.71$ and $\zeta_{6}^{\mathrm{L}} / \zeta_{2}^{\mathrm{L}}=2.16$. The insets show the logarithmic derivative $\mathrm{d} \log \left\langle\left[V^{\|}(t)\right]^{p}\right\rangle / \mathrm{d} \log \left\langle\left[V^{\|}(t)\right]^{2}\right\rangle$ for (a) $p=4$ and (b) $p=6$ as a function of $t / t_{0}$; there the bold dashed lines show the Lagrangian multifractal scaling and the thin lines what is expected from a self-similar behavior.

larger initial separations. The actual level of statistics do not allow us to relate systematically this behavior to that of the initial velocity difference distribution.

Finally another way to address the question of intermittency of the velocity difference consists in finding how moments of its longitudinal component depend on time. For that we follow, as in the case of the moments of distances, an approach similar to that of extended self-similarity. Figures 12 (a) and (b) show the fourth and sixth-order moments of $V^{\|}(t)$ as a function of its second-order moment. As evidenced in the insets, they display an anomalous behavior that differs from simple scaling. However, the collapse for various $r_{0}$ is much less evident than for the moments of the distance, except perhaps at sufficiently large times. One can there guess a power-law dependence of $\left\langle\left[V^{\|}(t)\right]^{p}\right\rangle$ as a function of $\left\langle\left[V^{\|}(t)\right]^{2}\right\rangle$. Surprisingly the power is compatible with the scaling exponent of the Lagrangian structure functions that were obtained in [25] by relating velocity increment along trajectories to She-Lévêque multifractal spectrum for the Eulerian field. The two dashed lines in Fig. 12 (a) and (b) correspond to the predicted values $\zeta_{4}^{\mathrm{L}} / \zeta_{2}^{\mathrm{L}}=1.71$ and $\zeta_{6}^{\mathrm{L}} / \zeta_{2}^{\mathrm{L}}=$ 2.16. Confirming further this match would require much better statistics.

\subsection{Stationary distribution of rescaled velocity differences}

As we have seen previously, the velocity difference between tracers displays very intermittent features and, as a consequence, does not converge to a behavior with temporal self-similarity, or does it only very slowly. The situation is very different when interested in mixed statistics between distances and longitudinal velocity differences. As seen in [20], the moment $\left\langle\left[V^{\|}(t)\right]^{3} /|\boldsymbol{R}(t)|\right\rangle$, which is initially negative and equal to $-(4 / 5) \epsilon$, tends very quickly to a positive constant - see Fig. 13 (a). The decrease at very large times comes from the contamination of the statistics by pairs that have reached a distance of the order of the integral scale. The asymptotic value $\approx 6.2 \epsilon$ seems to depend only weakly on the Reynolds number. The collapse of the curves associated to different Reynolds numbers and, for $r_{0} \gg \eta$, to various initial separations indicate that the time of convergence is $\propto t_{0}$. Figure 13 (b) shows the same moment but conditioned on the sign of the initial longitudinal velocity difference. One observes that for initially separating pairs (red curve), the convergence to the asymptotic value is on a time of the order of $\tau_{\eta}$. Conversely for tracers that initially approach each other (blue curve), the convergence is less fast. We have seen in Sec. 3.2 that such pairs first attain their minimal distance at 

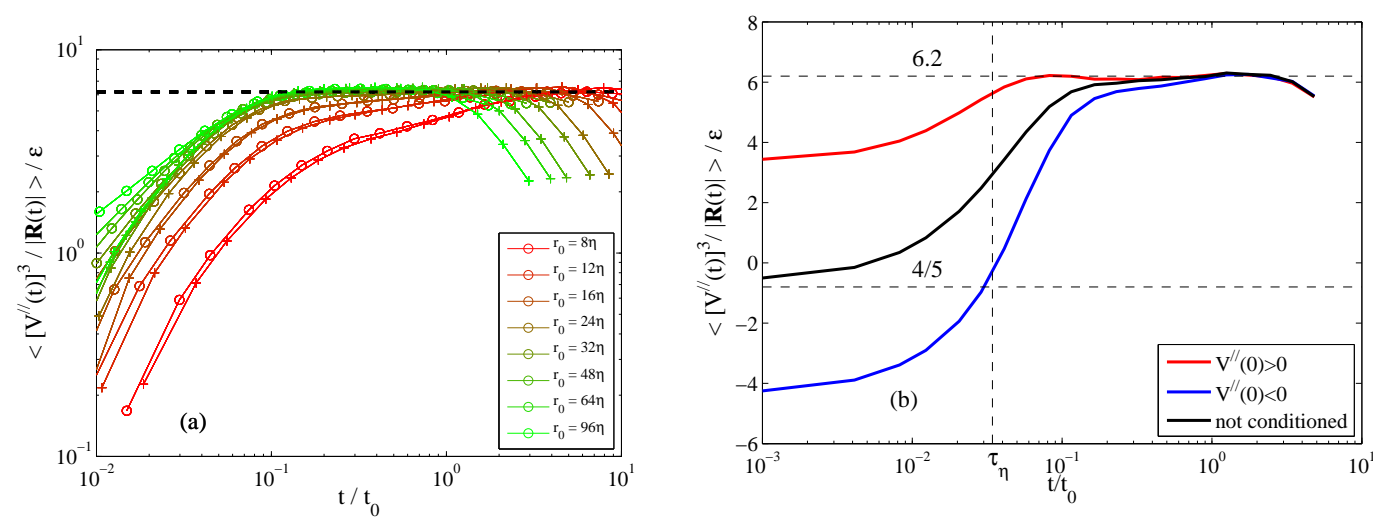

Figure 13. (a) Time evolution of the mixed moment $\left\langle\left[V^{\|}(t)\right]^{3} /|\boldsymbol{R}(t)|\right\rangle$ for different initial separations and the two values of the Reynolds number: $R_{\lambda}=730$ (o) and $R_{\lambda}=460(+)$. The black dashed line shows the asymptotic value $6.2 \epsilon$. (b) Same for $r_{0}=24 \eta$ and $R_{\lambda}=730$ in black and for the same moment but conditioned on positive (red curve) and negative (blue curve) values of the initial longitudinal velocity difference $V^{\|}(0)$.

$t \approx t_{*} \approx 0.01 t_{0}$. Then, at that time, $\left\langle\left[V^{\|}(t)\right]^{3} /|\boldsymbol{R}(t)| \mid V^{\|}(0)<0\right\rangle$ changes sign and the convergence to $\approx 6.2 \epsilon$ occurs only later. Such initially approaching pairs are leading the average so that the overall convergence is on timescales of the order of $t_{*}$. The mixed moment $\left\langle\left[V^{\|}\right]^{3} /|\boldsymbol{R}|\right\rangle$, which is a kind of "local dissipation" along pairs of trajectories, is thus conserved by the Lagrangian flow.

Actually, it is not only the average of the "local dissipation" that converges to a constant but its full distribution seems to attain a stationary regime on times of the order of $t_{*}$. Figure 14 (a) shows for a given initial separation, the convergence at large times of the PDF of $\left[V^{\|}(t)\right]^{3} /|\boldsymbol{R}(t)|$. One observes that the right and left tails converge on different timescales. The tail associated to large positive values (separating pairs) occurs on timescales of the order of $\tau_{\eta}$ (which is in this case $\approx 0.07 t_{0}$ ), while that for negative values (approaching pairs) converges slower. For the largest time $\left(t \approx 5 t_{0}\right)$ one observes that deviations to the asymptotic distribution occur again at very large positive values. This is due to a contamination of such events by the large scales of the turbulent flow. This decrease is in agreement with the observed departure in Fig. 13 of the average from its asymptotic value at large times. Figure 14 (b) shows the PDFs of $\left[V^{\|}\right]^{3} /|\boldsymbol{R}|$ for different initial separations and at a fixed time sufficiently large to be ensured that all distributions have attained their asymptotic regime. One observes a robust collapse, much more pronounced than for both the distribution of separations and that of velocity differences. Note
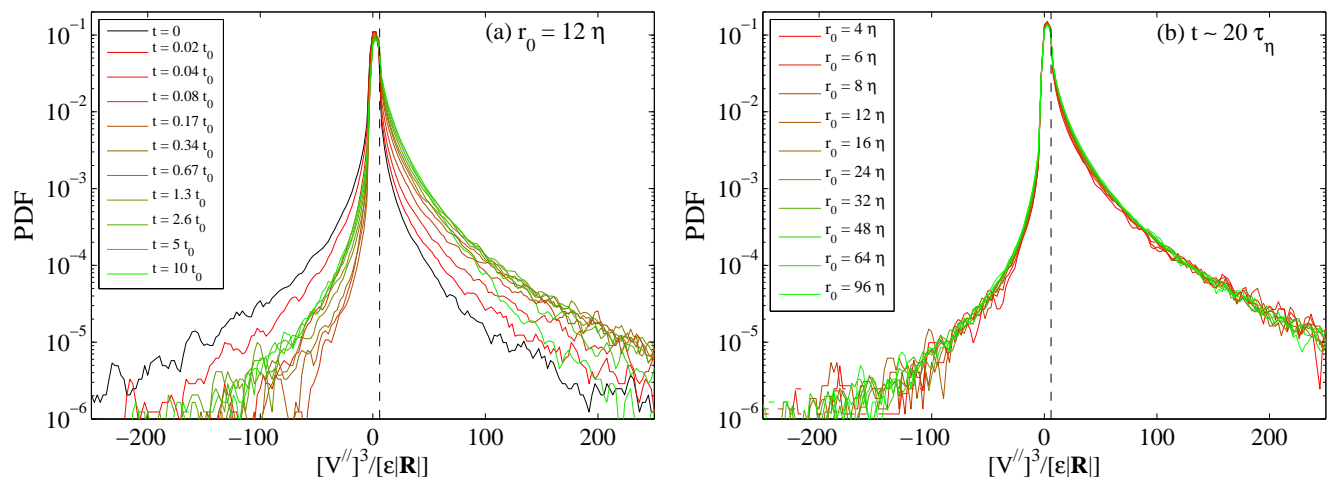

Figure 14. (a) PDF of the "local dissipation" $\left[V^{\|}(t)\right]^{3} /|\boldsymbol{R}(t)|$ for $r_{0}=24 \eta$ and various times (as labeled). (b) Same for various initial separations and a time $t=20 \tau_{\eta}$ fixed. $t / t_{0}$ goes here from 0.2 to 5 so that, in all cases, the mixed quantity $\left[V^{\|}\right]^{3} /|\boldsymbol{R}|$ has reached its asymptotic regime. In both figures the vertical dashed lines show the position of the average value $\approx 6.2 \epsilon$. 
that in Fig. 14 the distributions are raw and were not rescaled by any moment of $\left[V^{\|}\right]^{3} /|\boldsymbol{R}|$. The asymptotic PDF is peaked around zero (rather than its mean value) and displays fat tails that, according to our data, are $\propto \exp \left(-C\left|V^{\|}\right| /|\boldsymbol{R}|^{1 / 3}\right)$ on both sides.

To our knowledge, such a fast and manifest convergence of these mixed statistics has never been reported before. From a phenomenological viewpoint, one expects $V^{\|} \propto t \propto|\boldsymbol{R}|^{1 / 3}$ in the explosive Richardson-Obukhov regime, so that the "local dissipation" $\left[V^{\|}\right]^{3} /|\boldsymbol{R}|$ should become constant at sufficiently large times. However we have observed here that the convergence of this quantity to its asymptotic value occurs much faster and in a much more definite manner than the convergence of the statistics of $|\boldsymbol{R}|$ and $|\boldsymbol{V}|$ to their respective asymptotic forms. This indicates that the statistical stationarity of the "local dissipation" is more likely to be a cause rather than a consequence of Richardson-Obukhov explosive separation. At the moment we unfortunately lack a clear understanding of the physical mechanisms that are responsible for the observed behavior of $\left[V^{\|}\right]^{3} /|\boldsymbol{R}|$ and that could shed light on its relation to explosive separation.

\section{Extreme events in separation statistics}

In this section we turn back to the statistics of the distance $|\boldsymbol{R}(t)|$ between tracers. Our goal is to explain the mechanisms leading to very large or very small values of this distance in the light of the various observations made in previous sections. Considering the relative dispersion of tracers conditioned on their initial distance $r_{0}$ can be geometrically interpreted in terms of the time evolution of an initially spherical surface of radius $r_{0}$ that is centered on a reference trajectory. The transport of this surface by a turbulent flow is generally very complex. Incompressibility implies that the volume of the sphere is conserved but the velocity field roughness will be responsible for strong distorsions of its surface. This is represented in Fig. 15 that shows for $R_{\lambda}=460$ at three various times the shape defined by the instantaneous position of 60 trajectories that are all initially at a distance $24 \eta$ from a reference tracer. We can qualitatively deduce from these snapshots that the large
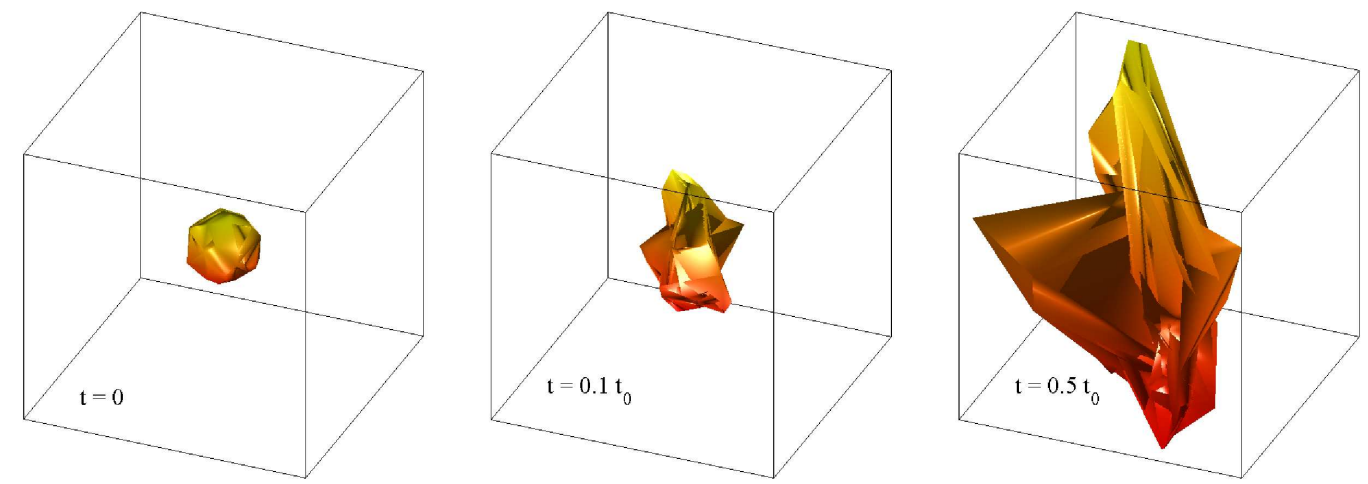

Figure 15. Quasi-Lagrangian evolution of the set of tracers that are initially at a distance $r_{0}=24 \eta$ of a reference trajectory.

excursions of the inter-trajectory distance go together with strong pinches of the surface. We also see that the intense stretchings are occurring in a time-correlated manner: the surface angles that are visible at late time have formed at very early stages. As we will now see, the most-separated pairs have been so for long times and carry a reminiscent dependence on the initial separation. 


\subsection{Memory in large-distance statistics}

We first consider the events related to distances that are much larger than their average. It was argued in Sec. 2.2 that the large-value tail of the separation PDF is not well described by Richardson's distribution. As seen in Fig. 6 for $t=5 t_{0}$, the tail is indeed broader than $\exp \left(-C|\boldsymbol{R}|^{2 / 3}\right)$ for $r_{0} \lesssim 8 \eta$ and narrower otherwise. It seems to tend to a Gaussian when either $r_{0}$ or $t$ are sufficiently large. We have checked that these behaviors are not due to a contamination by pairs that have reached the large scales of the turbulent flow, as the considered distances are still well below $L$, except maybe for the largest initial separation $r_{0}=24 \eta$. These observations suggest that for such extreme events, there is a long-term memory of the initial separation.

To qualify further the history of pairs that are well separated at large times, we have performed the following analysis. Fixing a final time $t_{\mathrm{f}}$ sufficiently large to have reached the explosive Richardson-Obukhov regime, we have carried out statistics conditioned on pairs that are far separated at $t=t_{\mathrm{f}}$, say such that their distance is $\left|\boldsymbol{R}\left(t_{\mathrm{f}}\right)\right| \geq 2\left\langle\left|\boldsymbol{R}\left(t_{\mathrm{f}}\right)\right|\right\rangle$. In order to not be contaminated by finite inertial subrange effects, we have restricted this analysis to $R_{\lambda}=730$ and to initial separations $2 \eta \leq r_{0} \leq 24 \eta$, and we have chosen the largest compatible value of $t_{\mathrm{f}}$, namely $t_{\mathrm{f}}=5 t_{0}$. Let us denote by $\langle\cdot\rangle_{+}$the resulting conditional ensemble average, i.e. $\langle\cdot\rangle_{+}=\left\langle\cdot \mid\left\{\left|\boldsymbol{R}\left(t_{\mathrm{f}}\right)\right| \geq\left\langle\left|\boldsymbol{R}\left(t_{\mathrm{f}}\right)\right|\right\rangle\right\}\right\rangle$. Figure 16 (a) represents the relative increase $\left\langle|\boldsymbol{R}(t)-\boldsymbol{R}(0)|^{2}\right\rangle_{+} /\left\langle|\boldsymbol{R}(t)-\boldsymbol{R}(0)|^{2}\right\rangle$ of the mean-squared displacement. The various curves, which are associated to different initial separations $r_{0}$, have a maximum at $t=t_{\mathrm{f}}=5 t_{0}$ represented by a dashed line. The value of this maximum is becoming larger when $r_{0}$ decreases and approaches the dissipative scales. This is a signature of the dependence of the large-positive value tail upon $r_{0}$. On the right-hand side of the maximum, the various curves tend back to 1 . This indicates that separations that are large at a given intermediate time relax in average to an ordinary behavior at later times. This can be regarded as a consequence of the weakening of largedistance probability tails as a function of time. The situation is rather different when interested in the left-hand side of the maximum. The curves do not converge to 1 , and thus to an average behavior when $t \rightarrow 0$. This means that pairs that are well separated at a given time are likely to have been so at any previous times. This asymmetry when going forward or backward in time could already be grasped in Fig. 15. The trajectories that are far away from the reference tracer at the latest
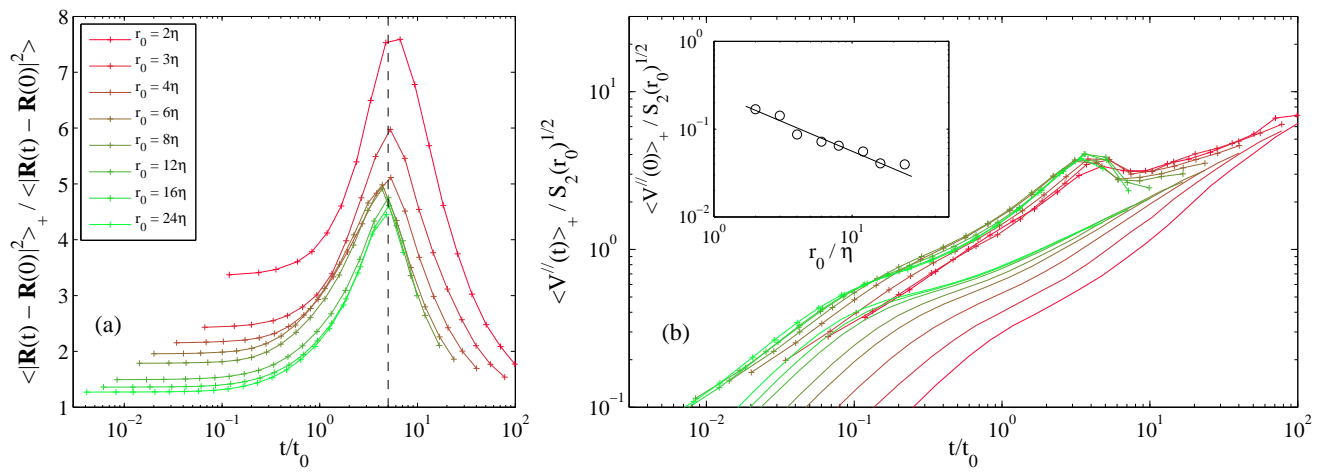

Figure 16. Conditional statistics over pairs that are at a distance at least twice the average at time $t_{\mathrm{f}}=5 t_{0}$ (conditional average on $\left|\boldsymbol{R}\left(5 t_{0}\right)\right| \geq 2\left\langle\left|\boldsymbol{R}\left(5 t_{0}\right)\right|\right\rangle$ is denoted by $\langle\cdot\rangle_{+}$). (a) Relative increase in the mean squared change of separation $\left\langle|\boldsymbol{R}(\bar{t})-\boldsymbol{R}(0)|^{2}\right\rangle$ as a function of time. (b) Averaged longitudinal velocity difference conditioned on the separation at time $t_{\mathrm{f}}=5 t_{0}(+)$ and without conditioning (solid line). Inset: initial value of the conditioned longitudinal velocity difference as a function of the initial separation; the solide line is $\propto r_{0}^{-2 / 3}$. 
time (as, e.g., those defining the left and top corners) are visibly also well separated at the intermediate time. Conversely the right corner at the intermediate time have later stopped separating faster than the average.

Figure 16 (b) shows the time evolution of the averaged longitudinal velocity difference $\left\langle V^{\|}(t)\right\rangle_{+}$conditioned on having a separation twice the average at time $t_{\mathrm{f}}=5 t_{0}$. A first remark that cannot be seen from the log-log plot is that the initial value of this average is strictly positive. The inset represents the variation of $\left\langle V^{\|}(0)\right\rangle_{+}$as a function of the initial separation. This implies that the pairs that are well separated at a late time are preferentially separating from the very beginning. The initial longitudinal velocity fluctuation that is necessary for the pairs to be far apart at time $t_{\mathrm{f}}$ becomes weaker when $r_{0}$ increases. Data suggest that $\left\langle V^{\|}(0)\right\rangle_{+} /\left[S_{2}\left(r_{0}\right)\right]^{1 / 2} \propto r_{0}^{-2 / 3}$ as seen in the inset. This initial separation makes the selected pairs reach an almost diffusive regime at time much shorter than the end of the average ballistic regime. For large-enough initial separations we indeed have $\left\langle V^{\|}(t)\right\rangle_{+} \sim t^{1 / 2}$ for $0.1 t_{0} \lesssim t \lesssim t_{0}$. The pair distance encountered a final acceleration right before the time of conditioning $t_{\mathrm{f}}$. After that, the longitudinal velocity difference relaxes slowly to the average regime. Again here, as in the case of the average separation, we observe that the imposing of having a large distance at a large time selects pair histories. The main contribution to statistics is indeed given by couples encountering an initially violent separation and quickly reaching larger scales. Such pairs converge rapidly to an explosive regime and continue to separate faster than the average for a long time. This is the snowball effect we have mentioned before.

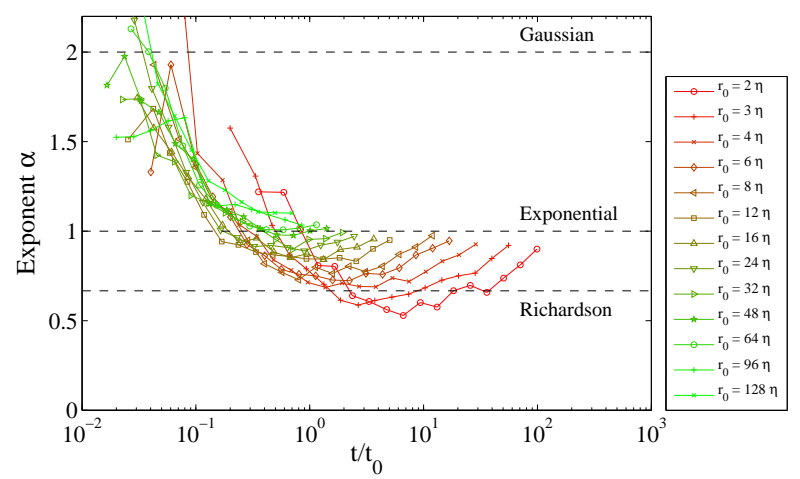

Figure 17. Exponent $\alpha$ of the right tail of the PDF of distances $|\boldsymbol{R}(t)|$ as a function of time and for various initial separations. The exponent was obtained by fitting $-\log p(|\boldsymbol{R}|)$ to a power-law for $\langle|\boldsymbol{R}(t)|\rangle<$ $|\boldsymbol{R}(t)|<0.5 L$.

In order to give a more quantitative handle on the far tail of the separation PDF, we have estimated and fitted its functional shape and studied its variations. For that, following the observations made in Sec. 2.2 , we have assumed that $p(|\boldsymbol{R}|) \propto$ $\exp \left(-C|\boldsymbol{R}|^{\alpha}\right)$ and measured how the exponent $\alpha$ depends on both time and initial separation. Figure 17] shows the time behavior of the exponent $\alpha$ for various values of the initial separation. On the figure, the three dashed horizontal lines represent the tails of a Gaussian $(\alpha=2)$, of an exponential $(\alpha=1)$ and of Richardson's distribution $(\alpha=2 / 3)$. At time $t=0$, the distribution is peaked around $r_{0}$ and compactly supported, so that $\alpha=\infty$. The exponent then decreases, crosses $\alpha=1$ (so that the distribution becomes a stretched exponential) for $t \approx 0.1 t_{0}$, and reaches a minimal value that depends on the initial separation $r_{0}$. For the smallest $r_{0}$, this minimal value is below Richardson's prediction, as previously noticed. For larger separations, this minimum increases and when $r_{0} \gg \eta$ the curves seem to saturate to the value $\alpha=1$. Also, we cannot exclude that all curves converge to 
the exponential value when $t \rightarrow \infty$. The increase at the last stage can hardly be blamed on an integral-scale effect. We have indeed excluded here all pairs that are separated by a distance larger than $L / 2$.

\section{2. "Fractal distribution" at small distances}
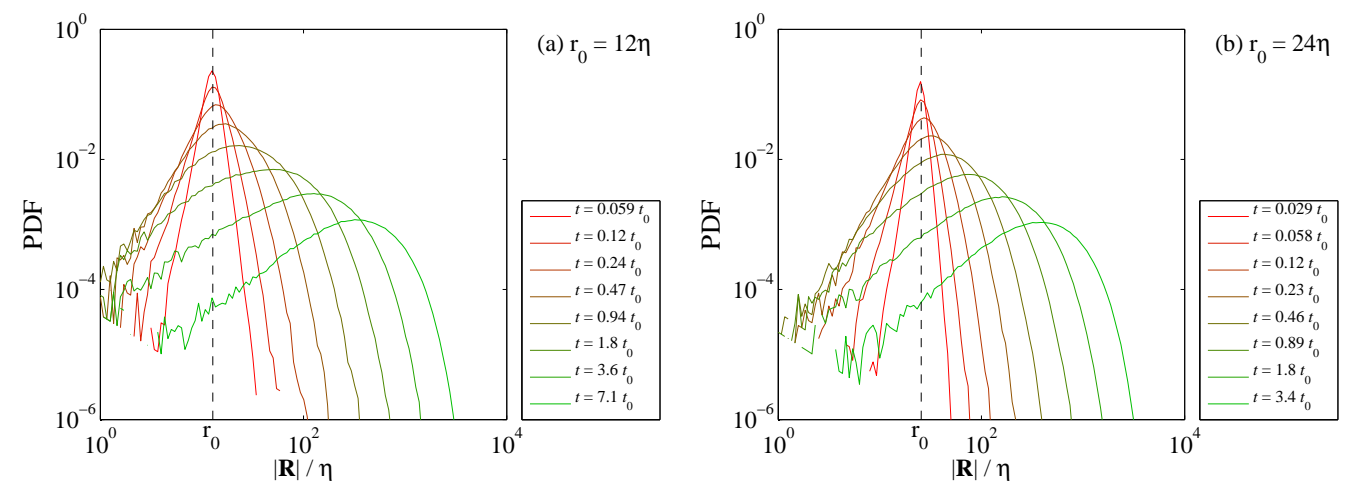

Figure 18. Unscaled PDFs of the absolute separation between tracers for various times and the initial separations (a) $r_{0}=12 \eta$ and (b) $r_{0}=24 \eta$.

Much more violent and intermittent events take place for particle pairs that separate much less than the average. As already stressed and observed in Fig. 15, the large excursions of inter-trajectory distances go together with strong pinches of separations. This is evidenced from Fig. 18 that represents the probability distribution of $|\boldsymbol{R}|$ at various times and for two inertial-range values of the initial separation. One observes that at very short times, the PDF is peaked around $r_{0}$. When time increases, its maximum, which roughly corresponds to the value of $\langle|\boldsymbol{R}(t)|\rangle$, shifts to larger values and the distribution broadens simultaneously at large and small values. This leads to the development for $r_{0} \ll|\boldsymbol{R}| \ll\langle|\boldsymbol{R}|\rangle$ of an intermediate range of pairs whose separations lag behind the average evolution. In this subrange, the PDF behaves as a power law $p(|\boldsymbol{R}|) \propto|\boldsymbol{R}|^{\beta}$, where the exponent $\beta$ evolves as a function of time and $r_{0}$. The power-law behavior is substantiated when measuring the cumulative probability $P^{<}(r)=\operatorname{Prob}[|\boldsymbol{R}|<r]=\int_{0}^{r} p\left(r^{\prime}\right) \mathrm{d} r^{\prime} \propto r^{\beta+1}$ of intertrajectory distances. One expects from Richardson arguments that $\beta=2$, so that $P^{<}(r) \propto r^{3}$.

The exponent $\beta$ can be interpreted in terms of fractal geometry. If all the trajectories were uniformly and independently distributed in space, the fraction of pairs at a distance less than $r$ would be $\propto r^{d}$, where $d=3$ is the space dimension. Also one would expect this fraction to be $\propto r^{d-1}$ if the trajectories were all confined on a surface, and $\propto r^{d-2}$ if they were on a curve. In general, the exponent $\beta+1=\lim _{r \rightarrow 0}\left[\log P^{<}(r)\right] /[\log r]$ measures the correlation dimension of a fractal set. Richardson's $r^{2}$ behavior thus corresponds to the idea that, at sufficiently long times, trajectories forget about their initial separation and distribute

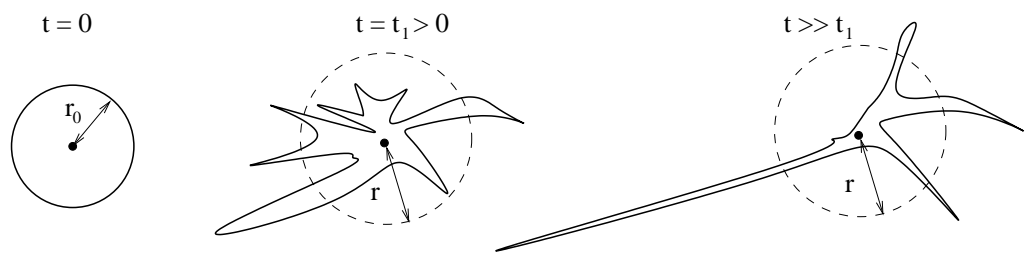

Figure 19. Two dimensional sketch of the time evolution of trajectories that are initially located at a distance $r_{0}$ of a reference tracer (shown as a black dot at the center of the circle) and then spread. 
homogeneously in space. As we have previously discussed, pair dispersion can be geometrically interpreted as the average Lagrangian evolution of an initially spherical surface of radius $r_{0}$ centered on a reference trajectory. At time $t=0$, it is clear that $P^{<}(r)$ is a Heaviside function centered on $r=r_{0}$, so that $\beta=\infty$. At later times the stretching and pinching of the sphere leads to a non-trivial behavior of $P^{<}(r)$ for $r \ll\langle|\boldsymbol{R}|\rangle$. This behavior is sketched for two dimensions in Fig. 19. The exponent $\beta$ is thus measuring the fractality of the image of the sphere by the Lagrangian flow. It relates to the fraction of this forward-in-time image that remains within a distance $r$ of the reference trajectory. At sufficiently large times (right-most panel of Fig. 19), one expects the set of trajectories to be mainly stretched by the flow eddies with the most relevant correlation time, that is those of size $\langle|\boldsymbol{R}(t)|\rangle$, and the small scale fluctuations to become less and less important. Hence, at very large times, the only pairs that are still at a distance $r \ll\langle|\boldsymbol{R}(t)|\rangle$ should be distributed on a surface (curve in two dimensions), so that $\beta+1 \simeq 2$ and $p(r) \propto r$. This approach contradicts the prediction of Richardson that, somehow, postulates that the velocity difference correlation time does not depend on scale (as it is assumed to be zero).
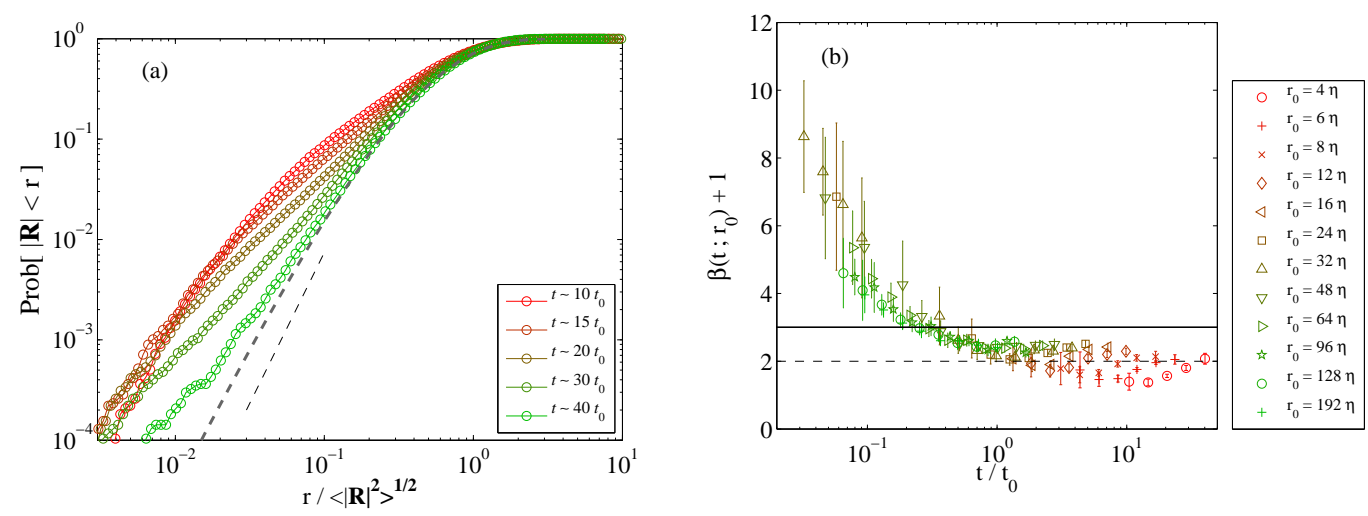

Figure 20. (a) Cumulative probability distribution of the distance $P^{<}(r)=\operatorname{Prob}[|\boldsymbol{R}|<r]$ as a function of $r$ for $r_{0}=4 \eta$ and at various times; the gray dashed curve is the cumulative probability associated to Richardson's distribution that displays a behavior $\propto r^{3}$ at small distances. (b) Time evolution of the left-tail power-law exponent $\beta$ for various values of the initial separation. The black solid line shows $\beta=2$ corresponding to Richardson's distribution and the dashed line stands for the value $\beta=1$ that is argued in the text.

To shed light on such ideas, we have estimated the exponent $\beta$ for various $r_{0}$ and at different times. For this purpose, we have used the cumulative probability $P^{<}(r)$ (shown in Fig. 20 (a) for $r_{0}=4 \eta$ ) to obtain $\beta+1$ through a fit of its local slope. The resulting measurements are displayed in Fig. 20 (b) as a function of time. Up to the large error bars due to a lack of statistics, one observes that the timescale $t_{0}$ is again relevant to describe both the initial dynamics and the convergence to an asymptotic regime. At small times $t \ll t_{0}$, the exponent $\beta$ takes large positive values, which, as explained above, are due to the initial conditioning $|\boldsymbol{R}(t)|=r_{0}$. At time $t \approx 0.3 t_{0}$, the exponent becomes smaller than 3 . For $t \gg t_{0}$, it asymptotically approaches the value $\beta=2$, so that data give evidence in favor of the argument exposed above. Interestingly, for small initial separations $r_{0} \lesssim 16 \eta$, the limit is reached from below and preceded by a minimum of $\beta$. This means that for such intermediate times, the pinching (or equivalently the stretching) of the sphere is so strong that the intersection of the latter with spheres of radius $r$ defines a fractal object that is more concentrated than a surface. Such a temporary singular behavior is certainly related to the presence of tails fatter than exponential at very large distances. 


\section{Concluding remarks}

In this paper we have confirmed and extended previous results of [20] on the timescales of convergence of turbulent pair dispersion to an asymptotic regime. We have seen that low and medium-order moments of the particle separation approach an asymptotic regime on times of the order of $t_{0}=S_{2}\left(r_{0}\right) /(2 \epsilon)$, where $S_{2}\left(r_{0}\right)$ denotes the (absolute value) structure function associated to the initial separation $r_{0}$ and $\epsilon$ is the mean rate of kinetic energy dissipation. This timescale has been shown to be relevant to describe also the initial kinematic change of sign of the longitudinal velocity difference $V^{\|}$and the tails of the distance distribution at small values. However, we have seen that $t_{0}$ is not relevant to describe the convergence of the velocity difference statistics to an asymptotic regime. We have observed that up to the largest time, the skewness, the flatness, and more generally the shape of the velocity difference distribution still depend on the initial separation, even when time is rescaled by $t_{0}$. This leads to a behavior that is by far more intermittent than that of separations. Also we obtained evidence that the far tail distribution of separation is also keeping a nontrivial memory on $r_{0}$ up to the largest times. Throughout this paper, we made an important use of geometrical considerations to explain phenomenologically the statistical events leading to extreme fluctuations. In particular, we argued and obtained numerical evidence that, at sufficiently large times, the probability distribution $p(r)$ of inter-tracer distances is $\propto r$ for $r \ll\langle|\boldsymbol{R}|\rangle$ and decays as $\exp (-C r)$ when $r \gg\langle|\boldsymbol{R}|\rangle$. These two observations strongly contradicts Richardson's eddy diffusivity approach, which seems nevertheless to give a good approximation of the core of the distribution. Finally, we obtained a striking result concerning mixed distance and velocity statistics. We indeed found that the distribution of a "local dissipation", defined as $\left[V^{\|}\right]^{3} /|\boldsymbol{R}|$, attains relatively quickly a scaling regime that is independent of both $r_{0}$ and time. This behavior, which, to our knowledge, has never been observed before, gives very strong constraints for the development and validation of stochastic Markovian models for turbulent relative dispersion.

A central question that we raised concerns the physical mechanisms leading to this fast convergence of the "local dissipation" to a statistically stationary behavior. At the moment, an even phenomenological explanation is still missing. We have understood that kinematic considerations can be used to explain why $\left\langle\left[V^{\|}\right]^{3} /|\boldsymbol{R}|\right\rangle$, which is initially negative, becomes quickly positive. This is due to the fact that initially approaching trajectories will always eventually separate. However, there is no clear idea of why this average should converge to a time-independent value. Possible ideas could consist in finding other mixed moments that are strictly (and not asymptotically) conserved by the two-point turbulent Lagrangian flow. Such considerations are kept for future work. Finally, another possible extension of the current study is to apply the developed understanding of the geometry of relative dispersion to more complicated turbulent transport situations involving more than two trajectories. This is for instance the case for the forward-in-time dynamics of triangles or tetrahedrons studied in [26]. The quality of today numerical data would be very useful to revisit such questions with an emphasize on extreme events. Another situation that is much closer to applications is that of the transport by turbulence of pollutant patches. We have seen in the current study that relative dispersion relates to the distorsion of a sphere by the Lagrangian flow. We related the small-scale behavior of separation statistics to the fractal dimension of this object. Such geometrical considerations could be generalized to understand and quantify the large concentration fluctuations in the dispersion of a pollutant spot. 


\section{Acknowledgments}

We are grateful to L. Biferale, M. Cencini, G. Falkovich, A. Frishmann, G. Krstulovic, A. Lanotte, S.S. Ray for useful discussions and remarks. Access to the IBM BlueGene/P computer JUGENE at the FZ Jülich was made available through the XXL-project HBO28. The research leading to these results has received funding from DFG-FOR1048 and from the European Research Council under the European Community's Seventh Framework Program (FP7/2007-2013, Grant Agreement no. 240579).

\section{References}

[1] G. Taylor, Diffusion by continuous movements, Proc. London Math. Soc. 20 (1921), pp. 196-212.

[2] A. Monin, and A. Yaglom Statistical Fluid Mechanics: Mechanics of Turbulence, Vol. 1, MIT press, Cambridge, USA, 1971.

[3] L. Richardson, Atmospheric diffusion shown on a distance-neighbour graph, Proc. R. Soc. Lond. Ser. A 110 (1926), pp. 709-737.

[4] A. Obukhov, On the distribution of energy in the spectrum of turbulent flow, Izv. Akad. Nauk SSSR, Ser. Geogr. Geofiz. 5 (1941), p. 453466.

[5] G. Falkovich, K. Gawędzki, and M. Vergassola, Particles and fields in fluid turbulence, Rev. Mod. Phys. 73 (2001), pp. 913-975.

[6] S. Ott, and J. Mann, An experimental investigation of the relative diffusion of particle pairs in three-dimensional turbulent flow, J. Fluid Mech. 422 (2000), pp. 207-223.

[7] L. Biferale, G. Boffetta, A. Celani, B. Devenish, A. Lanotte, and F. Toschi, Lagrangian statistics of particle pairs in homogeneous isotropic turbulence, Phys. Fluids 17 (2005), p. 115101.

[8] N. Ouellette, H. Xu, M. Bourgoin, and E. Bodenschatz, An experimental study of turbulent relative dispersion models, New J. Phys. 8 (2006), p. 109.

[9] J. Salazar, and L. Collins, Two-Particle Dispersion in Isotropic Turbulent Flows, Annu. Rev. Fluid Mech. 41 (2009), pp. 405-432.

[10] G.L. Eyink, Stochastic flux freezing and magnetic dynamo, Phys. Rev. E 83 (2011), p. 056405.

[11] G. Batchelor, Diffusion in a field of homogeneous turbulence. II The relative motion of particles, Proc. Camb. Phil. Soc. 48 (1952), pp. 345-363.

[12] R. Kraichnan, Dispersion of particle pairs in homogeneous turbulence, Phys. Fluids 9 (1966), p. 1937.

[13] R. Scatamacchia, L. Biferale, and F. Toschi, Extreme events in the dispersions of two neighboring particles under the influence of fluid turbulence, (2012).

[14] G. Batchelor, The application of the similarity theory of turbulence to atmospheric diffusion, Quart. J. R. Meteorol. Soc. 76 (1950), p. 133146.

[15] O. Kurbanmuradov, Stochastic Lagrangian models for two-particle trelative turbulent dispersion in high-Reynolds number turbulence, Monte Carlo Methods and Appl. 3 (1997), pp. 37-52.

[16] B. Sawford, Turbulent relative dispersion, Annu. Rev. Fluid Mech. 33 (2001), pp. 289-317.

[17] D.J.Thomson, Criteria for the selection of stochastic models of particle trajectories in turbulent flows, J. Fluid Mech. 180 (1987), pp. 529-556.

[18] M. Borgas, and P. Yeung, Relative dispersion in isotropic turbulence. Part 2. A new stochastic model with Reynolds-number dependence, J. Fluid Mech. 503 (2004), pp. 125-160.

[19] U. Frisch Turbulence, Cambridge University Press, Cambridge, UK, 1996.

[20] R. Bitane, J. Bec, and H. Homann, Timescales of turbulent relative dispersion, (2012).

[21] F. Toschi, and E. Bodenschatz, Lagrangian properties of particles in turbulence, Ann. Rev. Fluid Mech. 41 (2009), pp. 375-404.

[22] R. Grauer, H. Homann, and J.F. Pinton, Longitudinal and Transverse structure functions in high Reynolds-number turbulence, New J. Phys. 14 (2012), p. 063016.

[23] G. Boffetta, and I. Sokolov, Relative Dispersion in Fully Developed Turbulence: The Richardson's Law and Intermittency Corrections, Phys. Rev. Lett. 88 (2002), p. 094501.

[24] P. Yeung, and M. Borgas, Relative dispersion in isotropic turbulence. Part 1. Direct numerical simulations and Reynolds-number dependence, J. Fluid Mech. 503 (2004), pp. 92-124.

[25] L. Biferale, G. Boffetta, A. Celani, B. Devenish, A. Lanotte, and F. Toschi, Multifractal statistics of Lagrangian velocity and acceleration in turbulence, Phys. Rev. Lett. 93 (2004), p. 064502.

[26] A. Pumir, B. Shraiman, and M. Chertkov, Geometry of Lagrangian Dispersion in Turbulence, Phys. Rev. Lett. 85 (2000), pp. 5324-5327. 